\title{
Pair production of the lightest chargino via gluon-gluon collisions *
}

\author{
Ma Wen-Gan ${ }^{a, b, c}$, Du Fei ${ }^{b}$, Zhou Mian-Lai $^{b}$, Jiang $\mathrm{Yi}^{b}, \mathrm{Han} \mathrm{Liang}^{b}$ and Zhou Hong ${ }^{b}$ \\ ${ }^{a}$ CCAST (World Laboratory), P.O.Box 8730, Beijing 100080, China. \\ ${ }^{b}$ Department of Modern Physics, University of Science and Technology \\ of China (USTC), Hefei, Anhui 230027, China. \\ ${ }^{c}$ Institute of Theoretical Physics, Academia Sinica, \\ P.O.Box 2735, Beijing 100080, China.
}

${ }^{*}$ The project supported by National Natural Science Foundation of China 


\begin{abstract}
The production of the lightest chargino pair from gluon-gluon fusion is studied in the minimal supersymmetric model(MSSM) at protonproton colliders. We find that with the chosen parameters, the production rate of the subprocess can be over 2.7 femto barn when the chargino is higgsino-like, and the corresponding total cross section in proton-proton collider can reach 56 femto barn at the LHC in the CPconserving MSSM. It shows that this loop mediated subprocess can be competitive with the standard Drell-Yan subprocess in proton-proton colliders, especially at the LHC. Furthermore, our calculation shows it would be possible to extract information about some CP-violating phase parameters, if we collected enough chargino pair events.
\end{abstract}

PACS number(s): 14.80.Ly, 12.15.Ji, 12.60.Jv 


\section{Introduction}

The Minimal Standard Model(MSM) [1] [2] is a successful theory of strong and electroweak interactions up to the present accessible energies. Only the symmetric breaking sector of the electroweak interactions remains to be directly tested by experiments. The multi-TeV Large Hadron Collider LHC at CERN and the possible future Next Linear Collider(NLC) are elaborately designed in order to detect the symmetry-breaking mechanism and new physics beyond the MSM. At present, the supersymmetric extended model(SUSY) [3] [0] is widely considered as the theoretically most appealing extension of the MSM. Apart from describing the experimental data as well as the MSM does, the supersymmetric theory is able to solve various theoretical problems, such as the fact that the SUSY model may provide an elegant way to solve the deficiencies like the huge hierarchy problem, the necessity of fine tuning and the non-occurrence of gauge coupling unification at high energies.

Searching for SUSY particles directly in experiment is one of the promising tasks in the present and future colliders. The accurate measurements of the sparticle production processes can give us much information about the MSSM[5]. Among various processes involving sparticles, chargino pair production is one of the most important reference processes of the MSSM which may appear firstly in $e^{+} e^{-}, \gamma \gamma$ and hadron colliders. The analyses treating chargino pair production at the theoretical level are shown in references [6] [7] [8] [9] [10]. So far no experimental evidence for charginos has been found at LEP2, and only the lower bound on the lightest chargino mass $m_{\tilde{\chi}_{1}^{ \pm}}$is given. Recent experimental reports show that the mass of lightest chargino may be larger than 90GeV [11] [12] 13] [14, and this bound 
depends mainly on the sneutrino mass and the mass difference between the chargino and the lightest SUSY particles(LSP) theoretically.

The precise measurements of chargino pair production rates and chargino masses give the possibilities of measuring some gaugino, higgsino couplings and constraining the mass scale of squarks, which might not be in direct reach in colliders. Several mechanisms can induce the production of chargino pair at $p p$ colliders. One is through the Drell-Yan mechanism of quark and antiquark, and another is by gluon-gluon fusion. Although the chargino pair production via gluon-gluon fusion is a one-loop process, the production rate can be significant due to the large gluon luminosity in hadron colliders. In this paper we concentrate on the capability of the lightest chargino pair production via gluon-gluon collisions at $p p$ colliders in frame of the MSSM with full one-loop Feynman diagrams. The paper is organized as follows: In section II, we introduce some features of the model concerning in this work. In section III we present the analytical expressions of the cross section for the subprocess $g g \rightarrow \tilde{\chi}_{1}^{+} \tilde{\chi}_{1}^{-}$. In section IV, we study the numerical results of the cross sections both for subprocess and parent process. Finally, a short summary is presented. In the Appendix, the relevant Feynman rules and some lengthy expressions of the form factors appearing in the cross section in section III are listed.

\section{The relevant theory of the MSSM.}

\section{A. The chargino-sector of the MSSM.}

In the MSSM theory the physical chargino mass eigenstates $\tilde{\chi}_{1,2}^{ \pm}$are the combinations of 
the charged gauginos and higgsinos. Their physical masses can be obtained by diagonalizing the corresponding mass matrix $X[3$. In the CP-noninvariant MSSM theory, the mass term for charginos in lagrangian is

$$
\begin{gathered}
-\mathcal{L}_{m}=\sum_{i} \tilde{\tilde{\chi}}_{i}^{+}\left(U^{*} X V^{-1}\right)_{i i} \tilde{\chi}_{i}^{+} \\
X=\left(\begin{array}{ll}
M_{S U(2)} & m_{W} \sqrt{2} \sin \beta \\
m_{W} \sqrt{2} \cos \beta & |\mu| e^{i \phi_{\mu}}
\end{array}\right),
\end{gathered}
$$

The complex phase of gaugino mass parameter $M_{S U(2)}$ can be rotated away by field transformation, so we set $M_{S U(2)}$ to be real. $\mu$ is the higgsino mass parameter. The $\mathrm{U}, \mathrm{V}$ are two $2 \times 2$ unitary matrices defined to diagonalize the matrix $X$ to a diagonal matrix $X_{D}$, namely,

$$
U^{*} X V^{\dagger}=X_{D}
$$

where $X_{D}$ has non-negative entries. The two diagonal elements of this matrix can be expressed in a general form as 9

$$
\begin{aligned}
M_{ \pm}^{2}= & \frac{1}{2}\left\{M_{S U(2)}^{2}+|\mu|^{2}+2 m_{W}^{2} \pm\left[\left(M_{S U(2)}^{2}-|\mu|^{2}\right)^{2}+4 m_{W}^{4} \cos ^{2} 2 \beta+\right.\right. \\
& \left.\left.4 m_{W}^{2}\left(M_{S U(2)}^{2}+|\mu|^{2}+2 M_{S U(2)}|\mu| \sin 2 \beta \cos \phi_{\mu}\right)\right]^{1 / 2}\right\}
\end{aligned}
$$

which just stands for the expression of masses of charginos $\tilde{\chi}_{1}^{ \pm}$and $\tilde{\chi}_{2}^{ \pm}$. Inverting equation (2.4.1), the fundamental SUSY parameters $M_{S U(2)}$ and $|\mu|$ can be obtained from the alternative expressions on the right-hand side of the following equations, respectively [9].

$$
\left(M_{S U(2)},|\mu|\right)=\frac{1}{2}\left(\sqrt{m_{\tilde{\chi}_{1}^{+}}^{2}+m_{\tilde{\chi}_{2}^{+}}^{2}-2 m_{W}^{2}+2 M_{c}^{2}} \pm \sqrt{m_{\tilde{\chi}_{1}^{+}}^{2}+m_{\tilde{\chi}_{2}^{+}}^{2}-2 m_{W}^{2}-2 M_{c}^{2}}\right),
$$


where

$$
M_{c}^{2}=m_{W}^{2} \cos \phi_{\mu} \sin 2 \beta+\sqrt{m_{\tilde{\chi}_{1}^{+}}^{2} m_{\tilde{\chi}_{2}^{+}}^{2}-m_{W}^{4} \sin ^{2} 2 \beta \sin ^{2} \phi_{\mu}}
$$

The diagonalizing matrices $\mathrm{U}$ and $\mathrm{V}$ are dependent on the complex phase of $\mu$ and can be written in a general form as

$$
\begin{aligned}
U & =\left(\begin{array}{ll}
\cos \theta_{U} e^{i\left(\phi_{1}+\xi_{1}\right)} & \sin \theta_{U} e^{i\left(\phi_{1}+\xi_{1}+\delta_{U}\right)} \\
-\sin \theta_{U} e^{i\left(\phi_{2}+\xi_{2}-\delta_{U}\right)} & \cos \theta_{U} e^{i\left(\phi_{2}+\xi_{2}\right)}
\end{array}\right) \\
V & =\left(\begin{array}{ll}
\cos \theta_{V} e^{i\left(\phi_{1}-\xi_{1}\right)} & \sin \theta_{V} e^{i\left(\phi_{1}-\xi_{1}+\delta_{V}\right)} \\
-\sin \theta_{V} e^{i\left(\phi_{2}-\xi_{2}-\delta_{V}\right)} & \cos \theta_{V} e^{i\left(\phi_{2}-\xi_{2}\right)}
\end{array}\right),
\end{aligned}
$$

In above equations the $\xi_{1}$ and $\xi_{2}$ are arbitrarily chosen phases. It indicates the matrices $\mathrm{U}$ and V satisfying Eq.(2.3) are not unique, namely, some arbitrary phases may be introduced into the physical fields. But our analysis shows that they have no effects on the CP-odd observables. The explicit forms of the other mixing and phase angles depending on the Lagrangian parameters are given as 15]

$$
\begin{gathered}
\tan \theta_{U}=\sqrt{\frac{M_{+}^{2}-M_{S U(2)}^{2}-2 m_{W}^{2} \sin ^{2} \beta}{M_{+}^{2}-|\mu|^{2}-2 m_{W}^{2} \cos ^{2} \beta}}, \\
\tan \theta_{V}=\sqrt{\frac{M_{+}^{2}-M_{S U(2)}^{2}-2 m_{W}^{2} \cos ^{2} \beta}{M_{+}^{2}-|\mu|^{2}-2 m_{W}^{2} \sin ^{2} \beta}}, \\
e^{i 2 \phi_{1}}=\frac{\cos \theta_{U}}{\cos \theta_{V}} \cdot \frac{M_{+}^{2}+M_{S U(2)}|\mu| \tan \beta e^{i \phi_{\mu}}-2 m_{W}^{2} \sin ^{2} \beta}{M_{+}\left(M_{S U(2)}+|\mu| \tan \beta e^{i \phi_{\mu}}\right)} \\
e^{i 2 \phi_{2}}=\frac{\cos \theta_{V}}{\cos \theta_{U}} \cdot \frac{M_{-}^{2}+M_{S U(2)}|\mu| \tan \beta e^{i \phi_{\mu}}-2 m_{W}^{2} \sin ^{2} \beta}{M_{-}\left(M_{S U(2)} \tan \beta+|\mu| e^{-i \phi_{\mu}}\right)} \\
e^{i \delta_{U}}=\frac{M_{S U(2)}+|\mu| e^{i \phi_{\mu}} \tan \beta}{\left|M_{S U(2)}+\right| \mu\left|e^{i \phi_{\mu}} \tan \beta\right|} \\
e^{i \delta_{V}}=\frac{M_{S U(2)} \tan \beta+|\mu| e^{i \phi_{\mu}}}{\left|M_{S U(2)} \tan \beta+\right| \mu \mid e^{i \phi_{\mu} \mid}}
\end{gathered}
$$




\section{B. The squark-sector of the MSSM.}

In the frame of the MSSM every quark has two scalar partners, the squarks $\tilde{q}_{L}$ and $\tilde{q}_{R}$. If there is no left-right squark mixing in the squark-sector, the mass matrix of a scalar quark including CP-odd phases in the mass term of lagrangian, takes the following form 24]:

$$
-\mathcal{L}_{m}=\left(\begin{array}{cc}
\tilde{q}_{L}^{*} & \tilde{q}_{R}^{*}
\end{array}\right)\left(\begin{array}{ll}
m_{\tilde{q}_{L}}^{2} & a_{q} m_{q} \\
a_{q}^{*} m_{q} & m_{\tilde{q}_{R}}^{2}
\end{array}\right)\left(\begin{array}{c}
\tilde{q}_{L} \\
\tilde{q}_{R}
\end{array}\right)
$$

where $\tilde{q}_{L}$ and $\tilde{q}_{R}$ are the current eigenstates and for the up-type scalar quarks, we have

$$
\begin{gathered}
m_{\tilde{q}_{L}}^{2}=\tilde{M}_{Q}^{2}+m_{q}^{2}+m_{Z}^{2}\left(\frac{1}{2}-Q_{q} s_{W}^{2}\right) \cos 2 \beta, \\
m_{\tilde{q}_{R}}^{2}=\tilde{M}_{U}^{2}+m_{q}^{2}+Q_{q} m_{Z}^{2} s_{W}^{2} \cos 2 \beta \\
a_{q}=\left|a_{q}\right| e^{-2 i \phi_{q}}=\mu \cot \beta+A_{q}^{*} \tilde{M} .
\end{gathered}
$$

For the down-type scalar quarks,

$$
\begin{gathered}
m_{\tilde{q}_{L}}^{2}=\tilde{M}_{Q}^{2}+m_{q}^{2}-m_{Z}^{2}\left(\frac{1}{2}+Q_{q} s_{W}^{2}\right) \cos 2 \beta, \\
m_{\tilde{q}_{R}}^{2}=\tilde{M}_{D}^{2}+m_{q}^{2}+Q_{q} m_{Z}^{2} s_{W}^{2} \cos 2 \beta, \\
a_{q}=\left|a_{q}\right| e^{-2 i \phi_{q}}=\mu \tan \beta+A_{q}^{*} \tilde{M},
\end{gathered}
$$

where $Q_{q}\left(Q_{D}=-\frac{1}{3}, Q_{U}=\frac{2}{3}\right)$ is the charge of the scalar quark, $\tilde{M}_{Q}^{2}, \tilde{M}_{U}^{2}$ and $\tilde{M}_{D}^{2}$ are the self-supersymmetry-breaking mass terms for the left-handed and right-handed scalar quarks, $s_{W}=\sin \theta_{W}, c_{W}=\sin \theta_{W}$. We choose $\tilde{M}_{Q}=\tilde{M}_{U}=\tilde{M}_{D}=\tilde{M} . A_{q} \cdot \tilde{M}$ is a trilinear scalar interaction parameter. The complex value $a_{q}$ can introduce CP-violation. In general, $\tilde{q}_{L}$ and 
$\tilde{q}_{R}$ are mixed and give the mass eigenstates $\tilde{q}_{1}$ and $\tilde{q}_{2}$ (usually we assume $m_{\tilde{q}_{1}}<m_{\tilde{q}_{2}}$ ). The mass eigenstates $\tilde{q}_{1}$ and $\tilde{q}_{2}$ are linear combinations of the current eigenstates $\tilde{q}_{L}, \tilde{q}_{R}$

$$
\begin{aligned}
& \tilde{q}_{1}=\tilde{q}_{L} \cos \theta_{q} e^{i \phi_{q}}-\tilde{q}_{R} \sin \theta_{q} e^{-i \phi_{q}}, \\
& \tilde{q}_{2}=\tilde{q}_{L} \sin \theta_{q} e^{i \phi_{q}}+\tilde{q}_{R} \cos \theta_{q} e^{-i \phi_{q}}
\end{aligned}
$$

and

$$
\tan 2 \theta_{q}=\frac{2\left|a_{q}\right| m_{q}}{m_{\tilde{q}_{L}}^{2}-m_{\tilde{q}_{R}}^{2}} .
$$

where the $\theta_{q}$ is the mixing angle and $\phi_{q}$ is the CP-violating phase. Then the masses of $\tilde{q}_{1}$ and $\tilde{q}_{2}$ are expressed as

$$
\left(m_{\tilde{q}_{1}}^{2}, m_{\tilde{q}_{2}}^{2}\right)=\frac{1}{2}\left\{m_{\tilde{q}_{L}}^{2}+m_{\tilde{q}_{R}}^{2} \mp\left[\left(m_{\tilde{q}_{L}}^{2}-m_{\tilde{q}_{R}}^{2}\right)^{2}+4\left|a_{q}\right|^{2} m_{q}^{2}\right]^{\frac{1}{2}}\right\} .
$$

In the CP-violating MSSM theory, there are several possibilities to introduce CP-odd phases 16]. In our process, two CP-odd phases are involved, respectively appearing in the squark mass and chargino mass matrices. The detailed analyses of the present upper bounds on electron and neutron electric dipole moments may give constraints on CP-odd phase parameters indirectly [17]. But these constraints should be rather weak, since those results depend strongly on the assumptions which are used. Recently S.Y. Choi et al discussed the impacts of the CP-odd phase stemming from chargino mass matrix in the production of lightest chargino-pair in $e^{+} e^{-}$collisions at the tree-level [18]. In reference [15] [9] the effects from the CP-odd phases in the processes of the top quark pair and lightest chargino pair productions in $\gamma \gamma$ collisions at one-loop level in frame of the CP-violating MSSM, are investigated. There 
all the relevant $\mathrm{CP}$-odd complex phases are kept and the $\mathrm{CP}$-violating effects are studied without any extra limitations on CP-odd phases for the general discussion. In this work we shall investigate the CP-odd effects in the same way as in previous works [15] [9].

\section{The Higgs-sector of the MSSM.}

The supersymmetric model requires at least the extension of one additional Higgs-doublet where the parameters of the Higgs sector are tightly related. In the MSSM the neutral Higgs boson masses $m_{h^{0}}, m_{H^{0}}$ and $m_{A^{0}}$ are given by 19

$$
\begin{aligned}
m_{h^{0}, H^{0}}^{2}= & \frac{1}{2}\left[m_{A^{0}}^{2}+m_{Z}^{2}+\epsilon \mp\right. \\
& \left.\sqrt{\left(m_{A^{0}}^{2}+m_{Z}^{2}+\epsilon\right)^{2}-4 m_{A^{0}}^{2} m_{Z}^{2} \cos ^{2} 2 \beta-4 \epsilon\left(m_{A^{0}}^{2} \sin ^{2} \beta+m_{Z}^{2} \cos ^{2} \beta\right)}\right] \\
& m_{H^{ \pm}}^{2}=m_{A^{0}}^{2}+m_{W}^{2}
\end{aligned}
$$

with the leading corrections being characterized by the radiative parameter $\epsilon$

$$
\epsilon=\frac{3 G_{F}}{\sqrt{2} \pi^{2}} \frac{m_{t}^{4}}{\sin ^{2} \beta} \log \left[\frac{m_{\tilde{t}}^{2}}{m_{t}^{2}}\right] .
$$

The parameter $m_{\tilde{t}}^{2}=m_{\tilde{t}_{1}} m_{\tilde{t}_{2}}$ denotes the average squared mass of the stop quarks. The mixing angle $\alpha$ is fixed by $\tan \beta$ and the Higgs boson mass $m_{A^{0}}$,

$$
\tan 2 \alpha=\tan 2 \beta \frac{m_{A^{0}}^{2}+m_{Z}^{2}}{m_{A^{0}}^{2}-m_{Z}^{2}+\frac{\epsilon}{\cos 2 \beta}}\left(-\frac{\pi}{2}<\alpha<0\right) .
$$

\section{The calculation of subprocess $g g \rightarrow \tilde{\chi}_{1}^{+} \tilde{\chi}_{1}^{-}$in the MSSM.}

The process producing the lightest chargino pair via gluon-gluon collisions can only be induced through one-loop diagrams. In this case it is not necessary to consider the renormalization at one-loop level and the ultraviolet divergence should be canceled automatically 
if all the one-loop diagrams in MSSM are included. In this work, we perform the evolution in the t'Hooft-Feynman gauge. The generic Feynman diagrams contributing to the subprocess $g g \rightarrow \tilde{\chi}_{1}^{+} \tilde{\chi}_{1}^{-}$in frame of the MSSM are depicted in figure 1, where the diagrams with exchanging incoming gluons are not shown except for quartic interaction diagrams shown in Fig.(b.1 2). The relevant Feynman rules can be found in Appendix A. All the one-loop diagrams can be divided into three groups: $(1)$ box diagrams shown in Fig.1(a.1 3). (2) quartic interaction diagrams in Fig.1(b.1 2). (3) triangle diagrams shown in Fig.1(c.1 2 ). In our calculation we find the contributions from the $\gamma$ (or $Z^{0}$ ) exchanging s-channel Feynman diagrams with quark loops as shown in Fig.1(c.2), are zero (or very small). It can be understood by the Furry theorem. The Furry theorem forbids the production of the spin-one components of the $Z^{0}$ and $\gamma$ from a fermion loop. And the contribution from the spin-zero component of the $Z^{0}$ vector boson coupling with a pair of charginos is very small. The contribution from each $\gamma\left(Z^{0}\right)$ exchanging s-channel diagram involving a squark loop shown in Fig.1(b.2) and Fig.1(c.1), is canceled out by the same type diagram, but involving its corresponding anti-squark loop. Here we should mention that there are also some diagrams having no contribution to the process, such as the s-channel diagrams with trilinear gluon interactions. Since the vertices of $A^{0}\left(G^{0}\right)-\tilde{q}_{i}-\tilde{q}_{i}$ vanish [4], there is no such diagram with a triangle squark loop coupling with $A^{0}$ or $G^{0}$ Higgs boson. All these diagrams are not drawn in Fig.1.

In this work, we denote the reaction of chargino-pair production via gluon-gluon collisions 
as:

$$
g\left(p_{3}, \mu\right) g\left(p_{4}, \nu\right) \longrightarrow \tilde{\chi}_{1}^{+}\left(p_{1}\right) \tilde{\chi}_{1}^{-}\left(p_{2}\right)
$$

We write the corresponding matrix element for each of the diagrams in Fig.1 in the form according to their Lorentz invariant structure:

$$
\begin{aligned}
& \delta \mathcal{M}=\mathcal{M}^{b}+\mathcal{M}^{t r}+\mathcal{M}^{q} \\
& =\mathcal{M}^{b, \hat{t}}+\mathcal{M}^{b, \hat{u}}+\mathcal{M}^{t r, \hat{t}}+\mathcal{M}^{t r, \hat{u}}+\mathcal{M}^{q} \\
& =\frac{1}{2} \delta_{a b} \epsilon^{\mu}\left(p_{3}\right) \epsilon^{\nu}\left(p_{4}\right) \bar{u}\left(p_{1}\right)\left\{f_{1} \gamma_{\mu} \gamma_{\nu}+f_{2} \gamma_{\nu} \gamma_{\mu}+f_{3} \gamma_{\mu} p_{1 \nu}+f_{4} \gamma_{\mu} p_{2 \nu}\right. \\
& +f_{5} \gamma_{\nu} p_{1 \mu}+f_{6} \gamma_{\nu} p_{2 \mu}+f_{7} p_{1 \mu} p_{1 \nu}+f_{8} p_{1 \mu} p_{2 \nu}+f_{9} p_{1 \nu} p_{2 \mu}+f_{10} p_{2 \mu} p_{2 \nu} \\
& +f_{11} \not p_{3} \gamma_{\mu} \gamma_{\nu}+f_{12} \not p_{3} \gamma_{\nu} \gamma_{\mu}+f_{13} p_{3} \gamma_{\mu} p_{1 \nu}+f_{14} p_{3} \gamma_{\mu} p_{2 \nu} \\
& +f_{15} \not p_{3} \gamma_{\nu} p_{1 \mu}+f_{16} \not p_{3} \gamma_{\nu} p_{2 \mu}+f_{17} p_{3} p_{1 \mu} p_{1 \nu}+f_{18} \not p_{3} p_{1 \mu} p_{2 \nu} \\
& +f_{19} p_{3} p_{1 \nu} p_{2 \mu}+f_{20} p_{3} p_{2 \mu} p_{2 \nu}+f_{21} \epsilon_{\mu \nu \alpha \beta} p_{1}^{\alpha} p_{3}^{\beta}+f_{22} \epsilon_{\mu \nu \alpha \beta} p_{2}^{\alpha} p_{3}^{\beta} \\
& +f_{23} \epsilon_{\mu \nu \alpha \gamma} p_{1}^{\alpha} \gamma^{\gamma}+f_{24} \epsilon_{\mu \nu \alpha \gamma} p_{2}^{\alpha} \gamma^{\gamma}+f_{25} \epsilon_{\mu \nu \alpha \gamma} p_{3}^{\alpha} \gamma^{\gamma}+f_{26} \epsilon_{\mu \alpha \beta \gamma} p_{1}^{\alpha} p_{3}^{\beta} \gamma^{\gamma} p_{1 \nu} \\
& +f_{27} \epsilon_{\mu \alpha \beta \gamma} p_{1}^{\alpha} p_{3}^{\beta} \gamma^{\gamma} p_{2 \nu}+f_{28} \epsilon_{\mu \alpha \beta \gamma} p_{2}^{\alpha} p_{3}^{\beta} \gamma^{\gamma} p_{1 \nu}+f_{29} \epsilon_{\mu \alpha \beta \gamma} p_{2}^{\alpha} p_{3}^{\beta} \gamma^{\gamma} p_{2 \nu} \\
& +f_{30} \epsilon_{\nu \alpha \beta \gamma} p_{1}^{\alpha} p_{3}^{\beta} \gamma^{\gamma} p_{1 \mu}+f_{31} \epsilon_{\nu \alpha \beta \gamma} p_{1}^{\alpha} p_{3}^{\beta} \gamma^{\gamma} p_{2 \mu}+f_{32} \epsilon_{\nu \alpha \beta \gamma} p_{2}^{\alpha} p_{3}^{\beta} \gamma^{\gamma} p_{1 \mu} \\
& +f_{33} \epsilon_{\nu \alpha \beta \gamma} p_{2}^{\alpha} p_{3}^{\beta} \gamma^{\gamma} p_{2 \mu}+f_{34} \gamma_{5} \gamma_{\mu} \gamma_{\nu}+f_{35} \gamma_{5} \gamma_{\nu} \gamma_{\mu} \\
& +f_{36} \gamma_{5} \gamma_{\mu} p_{1 \nu}+f_{37} \gamma_{5} \gamma_{\mu} p_{2 \nu}+f_{38} \gamma_{5} \gamma_{\nu} p_{1 \mu}+f_{39} \gamma_{5} \gamma_{\nu} p_{2 \mu} \\
& +f_{40} \gamma_{5} p_{1 \mu} p_{1 \nu}+f_{41} \gamma_{5} p_{1 \mu} p_{2 \nu}+f_{42} \gamma_{5} p_{1 \nu} p_{2 \mu}+f_{43} \gamma_{5} p_{2 \mu} p_{2 \nu}+f_{44} \gamma_{5} p_{3} \gamma_{\mu} \gamma_{\nu} \\
& +f_{45} \gamma_{5} \not p_{3} \gamma_{\nu} \gamma_{\mu}+f_{46} \gamma_{5} p_{3} \gamma_{\mu} p_{1 \nu}+f_{47} \gamma_{5} p_{3} \gamma_{\mu} p_{2 \nu}+f_{48} \gamma_{5} p_{3} \gamma_{\nu} p_{1 \mu} \\
& +f_{49} \gamma_{5} \not p_{3} \gamma_{\nu} p_{2 \mu}+f_{50} \gamma_{5} p_{3} p_{1 \mu} p_{1 \nu}+f_{51} \gamma_{5} p_{3} p_{1 \mu} p_{2 \nu}+f_{52} \gamma_{5} p_{3} p_{1 \nu} p_{2 \mu}
\end{aligned}
$$




$$
\begin{aligned}
& +f_{53} \gamma_{5} p_{3} p_{2 \mu} p_{2 \nu}+f_{54} \epsilon_{\mu \nu \alpha \beta} p_{1}^{\alpha} p_{3}^{\beta} \gamma_{5}+f_{55} \epsilon_{\mu \nu \alpha \beta} p_{2}^{\alpha} p_{3}^{\beta} \gamma_{5}+f_{56} \epsilon_{\mu \nu \alpha \gamma} p_{1}^{\alpha} \gamma_{5} \gamma^{\gamma} \\
& +f_{57} \epsilon_{\mu \nu \alpha \gamma} p_{2}^{\alpha} \gamma_{5} \gamma^{\gamma}+f_{58} \epsilon_{\mu \nu \alpha \gamma} p_{3}^{\alpha} \gamma_{5} \gamma^{\gamma}+f_{59} \epsilon_{\mu \delta \alpha \gamma} p_{1}^{\delta} p_{3}^{\alpha} \gamma_{5} \gamma^{\gamma} p_{1 \nu}+f_{60} \epsilon_{\mu \delta \alpha \gamma} p_{1}^{\delta} p_{3}^{\alpha} \gamma_{5} \gamma^{\gamma} p_{2 \nu} \\
& +f_{61} \epsilon_{\mu \delta \alpha \gamma} p_{2}^{\delta} p_{3}^{\alpha} \gamma_{5} \gamma^{\gamma} p_{1 \nu}+f_{62} \epsilon_{\mu \delta \alpha \gamma} p_{2}^{\delta} p_{3}^{\alpha} \gamma_{5} \gamma^{\gamma} p_{2 \nu}+f_{63} \epsilon_{\nu \delta \alpha \gamma} p_{1}^{\delta} p_{3}^{\alpha} \gamma_{5} \gamma^{\gamma} p_{1 \mu} \\
& \left.+f_{64} \epsilon_{\nu \delta \alpha \gamma} p_{1}^{\delta} p_{3}^{\alpha} \gamma_{5} \gamma^{\gamma} p_{2 \mu}+f_{65} \epsilon_{\nu \delta \alpha \gamma} p_{2}^{\delta} p_{3}^{\alpha} \gamma_{5} \gamma^{\gamma} p_{1 \mu}+f_{66} \epsilon_{\nu \delta \alpha \gamma} p_{2}^{\delta} p_{3}^{\alpha} \gamma_{5} \gamma^{\gamma} p_{2 \mu}\right\} v\left(p_{2}\right),
\end{aligned}
$$

with

$$
f_{i}=f_{i}^{b}+f_{i}^{t r}+f_{i}^{q} \quad(i=1 \sim 66),
$$

where $\frac{1}{2} \delta_{a b}$ is the color factors in amplitudes, $\mathcal{M}^{b}, \mathcal{M}^{t r}$ and $\mathcal{M}^{q}$ are the matrix elements contributed by box, triangle and quartic interactions diagrams, respectively. $f_{i}^{b}, f_{i}^{t r}$ and $f_{i}^{q}$ are their corresponding form factors. As we divided the matrix elements $\mathcal{M}^{b}$ and $\mathcal{M}^{\text {tr }}$ into t- and u-channel parts, respectively, so for each of the corresponding form factors we have

$$
f_{i}^{b}=f_{i}^{b, \hat{t}}+f_{i}^{b, \hat{u}}, \quad f_{i}^{t r}=f_{i}^{t r, \hat{t}}+f_{i}^{t r, \hat{u}} \quad(i=1 \sim 66) .
$$

Since the amplitude parts from the u-channel box and triangle vertex interaction diagrams can be obtained from the t-channel's by doing exchanges shown as below:

$$
\mathcal{M}^{j, \hat{u}}=\mathcal{M}^{j, \hat{t}}\left(\hat{t} \rightarrow \hat{u}, p_{3} \leftrightarrow p_{4}, \mu \leftrightarrow \nu\right), \quad(j=b, t r)
$$

Then only the explicit t-channel form factors $f_{i}^{b, \hat{t}}$ and $f_{i}^{t r, \hat{t}}(i=1 \sim 66)$ for box and triangle diagrams, and the form factors for quartic interaction diagrams are listed in Appendix B. The cross section for this subprocess at the one-loop order in unpolarized photon collisions can be obtained by

$$
\hat{\sigma}(\hat{s})=\frac{1}{16 \pi \hat{s}^{2}} \int_{\hat{t}^{-}}^{\hat{t}^{+}} d \hat{t} \sum|\mathcal{M}|^{2}
$$


where $\hat{t}^{ \pm}=\left(m_{\tilde{\chi}_{1}^{+}}^{2}-\frac{1}{2} \hat{s}\right) \pm \frac{1}{2} \hat{s} \beta$. The bar over sum notation means that we are doing average over initial spins and colors.

\section{Numerical results and discussions}

In this section, we present some numerical results of the total cross section from the full one-loop diagrams involving virtual (s)quarks for the subprocess of $g g \rightarrow \tilde{\chi}_{1}^{+} \tilde{\chi}_{1}^{-}$and parent process $p p \rightarrow g g \rightarrow \tilde{\chi}_{1}^{+} \tilde{\chi}_{1}^{-}+X$, respectively. The general input parameters involved are chosen as: $m_{t}=175 \mathrm{GeV}, m_{Z}=91.187 \mathrm{GeV}, m_{b}=4.5 \mathrm{GeV}, \sin ^{2} \theta_{W}=0.2315$, and $\alpha=1 / 137.036$. We adopt a simple one-loop formula for the running strong coupling constant $\alpha_{s}$ as

$$
\alpha_{s}(\mu)=\frac{\alpha_{s}\left(m_{Z}\right)}{1+\frac{33-2 n_{f}}{6 \pi} \alpha_{s}\left(m_{Z}\right) \ln \left(\frac{\mu}{m_{Z}}\right)} .
$$

where $\alpha_{s}\left(m_{Z}\right)=0.117$ and $n_{f}$ is the number of active flavors at energy scale $\mu$.

We take the numerical values of the MSSM parameters in CP-conserving case which are also acceptable in the frame of the Minimal Supergravity (mSUGRA) model, since the mSUGRA is the simplest and most fully investigated model. It assumes that the boundary conditions are set at $M_{U}$. The ranges of the model parameters should be constrained by the evolution to low energies $\underset{\sim}{<} m_{S U S Y}$ beginning with the boundary conditions at $M_{U}$. With this consideration we take the following parameter values by default unless otherwise stated.

The squark masses of the first two generations are approximately degenerated, namely, we can neglect their mixing angles between the left- and right-squarks. and choose $m_{\tilde{u}_{1,2}}=$ $m_{\tilde{d}_{1,2}}=m_{\tilde{c}_{1,2}}=m_{\tilde{s}_{1,2}}=600 \mathrm{GeV}$ From renormalization group equations [21] one expects that 
the soft SUSY breaking masses $m_{\tilde{q}_{L}}$ and $m_{\tilde{q}_{R}}$ of the third generation squarks are smaller than those of the first and second generations due to the Yukawa interactions. The third family stop quarks are normally significantly mixed and split due to the large mass of the top quark, and the lightest scalar top quark mass eigenstate $\tilde{t}_{1}$ can be much lighter than the top quark and all the scalar partners of the light quarks. Therefore we assume $m_{\tilde{t}_{1}}<m_{\tilde{t}_{2}}$ and $\theta_{t} \sim \frac{\pi}{4}$, and take $\tilde{M}_{Q}=\tilde{M}_{U}=\tilde{M}_{D}=\tilde{M}=200 \mathrm{GeV}$ for the third generation squarks. For simplicity, we set the sbottom mixing angle being zero $\left(\theta_{b}=0\right)$. Then the masses of stop, sbottom mass eigenstates $\tilde{t}_{1,2}$ and $\tilde{b}_{1,2}$ can be determined quantitatively by Eqs.(2.8 $\sim 2.16$ ). For the CP-odd neutral Higgs mass $m_{A^{0}}$ we set its value being typical large and take $m_{A^{0}}=250 \mathrm{GeV}$. The ratio of the vacuum expectation values $\tan \beta$ is chosen to be 4 or 40 in order to make comparison. The masses of other Higgs bosons can be obtained from Eqs. $(2.17) \sim(2.20)$. We checked that with these input parameters the experimental limits on the masses of Higgs bosons are not violated. Since these mass values of Higgs bosons are far below the threshold of chargino pair production in our numerical calculation, we set all the decay widths of Higgs bosons to be $10 \mathrm{GeV}$ and these value choices will not influence our results significantly. In the CP-violating case, we use also the above input parameters for comparison.

The physical chargino masses $m_{\tilde{\chi}_{1}^{+}}$and $m_{\tilde{\chi}_{2}^{+}}$are taken to be $165 \mathrm{GeV}$ and $750 \mathrm{GeV}$, respectively. The fundamental SUSY parameters $M_{S U(2)}$ and $|\mu|$ can be extracted at the tree level from these input chargino masses, $\tan \beta$ and the complex phase angle of $\mu$ by using Eqs. $(2.4 .2 \sim 3)$. When $\mu$ is real, we assume $\mu$ is positive. Then the lightest chargino is 
dominantly gaugino (gaugino-like or wino-like) when there is $M_{S U(2)}<<\mu$, and the chargino is dominantly higgsino (higgsino-like), when $M_{S U(2)}$ is much larger than $\mu$. In the following we shall investigate the numerical results in both extreme cases.

The total cross sections of the lightest chargino pair production via gluon fusion as the functions of the c.m.s. energy of gluons $\sqrt{\hat{s}}$ with $m_{\tilde{\chi}_{1}^{+}}=165 \mathrm{GeV}, m_{\tilde{\chi}_{2}^{+}}=750 \mathrm{GeV}$ and all vanishing CP phases, are shown in Fig.2(a) and (b). In figure 2(a) the two curves correspond to the higgsino-like chargino case with $\tan \beta=4$ and $\tan \beta=40$, respectively. Whereas the plot in Figure 2(b) is for gaugino-like chargino case. It is obvious that the subprocess cross section of the pair production of the lightest higgsino-like chargino is one order larger than that of the gaugino-like chargino pair production. And in general, the cross sections with $\tan \beta=40$ are approximately one to four times larger than those with $\tan \beta=4$. Because of the resonance effects, all the four curves in Fig.2(a) $\sim(\mathrm{b})$ have peaks and spikes at the energy positions where the resonance conditions are satisfied. There are turn points on all four curves, which are located at the vicinity of $\sqrt{\hat{s}}=2 m_{t}=350 \mathrm{GeV}$. On the two curves of Fig.2(a,b) with $\tan \beta=4$, there are two small spikes stemming from resonance effects in the vicinities of $\sqrt{\hat{s}} \sim 2 m_{\tilde{b}_{1}} \sim 403 \mathrm{GeV}$ and $\sqrt{\hat{s}} \sim 2 m_{\tilde{b}_{2}} \sim 415 \mathrm{GeV}$, respectively. Whereas for the other two curves in Fig.2(a,b) with $\tan \beta=40$, the small spikes due to resonance effect are located at the positions of $\sqrt{\hat{s}} \sim 2 m_{\tilde{b}_{1}} \sim 403 \mathrm{GeV}$ and $\sqrt{\hat{s}}=2 m_{\tilde{b}_{2}} \sim 417 \mathrm{GeV}$, respectively.

Figure 3 gives the total cross sections of the subprocess as the functions of the lightest higgsino-like chargino mass with $\sqrt{\hat{s}}=450 \mathrm{GeV}$. In this figure we can see considerable enhancement around the region of $m_{\tilde{\chi}_{1}^{+}}=185 \mathrm{GeV}$. Figure 4 gives the cross sections of 
the higgsino-like chargino pair production subprocess as a function of self-supersymmetrybreaking mass parameter of the third generation scalar quarks $\tilde{M}$, when $\sqrt{\hat{s}}=450 G e V$ (Here we set the masses for squarks of the first and second generations, are degenerated and have the values being $600 \mathrm{GeV}$.). The two curves have obvious spikes due to resonance effect at the positions of $\tilde{M}=215 \mathrm{GeV}$ for both $\tan \beta=4$ and $\tan \beta=40$, respectively. There we have $\sqrt{\hat{s}}=450 G e V \sim 2 m_{\tilde{b}_{1,2}}$.

The cross sections in the subprocess of higgsino-like chargino pair production versus the CP phases angles $\phi_{C P}\left(=\phi_{\mu}, \phi_{q}\right)$ (Here we take $\phi_{q}=\phi_{t}=\phi_{b}$ and $\phi_{u, d, c, s}=0$ ) with $\sqrt{\hat{s}}=450 \mathrm{GeV}, m_{\tilde{\chi}_{1}^{+}}=165 \mathrm{GeV}$ and $m_{\tilde{\chi}_{2}^{+}}=750 \mathrm{GeV}$, are depicted in figure $5(\mathrm{a})$ and $5(\mathrm{~b})$. Fig.5(a) is for $\tan \beta=40$ and Fig.5(b) for $\tan \beta=4$. In both figures, the full-lines and dotted-lines correspond to $\phi_{C P}=\phi_{q}(q=t, b)$ and $\phi_{C P}=\phi_{\mu}$, respectively. The curves in Fig.5(a,b) show the periodical features of $\hat{\sigma}\left(\phi_{q}\right)=\hat{\sigma}\left(\pi+\phi_{q}\right)$ for the curves of $\hat{\sigma}$ versus $\phi_{q}$ and $\hat{\sigma}\left(\phi_{\mu}\right)=\hat{\sigma}\left(2 \pi+\phi_{\mu}\right)$ for the curves of $\hat{\sigma}$ versus $\phi_{\mu}$, respectively. All the two CP phase angles affect the cross sections obviously, but the effects from the phase angle $\phi_{q}$ are a little stronger than those from $\phi_{\mu}$.

With the chargino pair production rate in gluon-gluon fusion, we can easily obtain the total cross section in $p p$ collider, by folding the cross section of the subprocess $\hat{\sigma}\left(g g \rightarrow \tilde{\chi}_{1}^{+} \tilde{\chi}_{1}^{-}\right)$ with the gluon luminosity.

$$
\sigma\left(p p \rightarrow g g \rightarrow \tilde{\chi}_{1}^{+} \tilde{\chi}_{1}^{-}+X\right)=\int_{4 m_{\tilde{\chi}_{1}^{+}}^{2} / s}^{1} d \tau \frac{d L_{g g}}{d \tau} \hat{\sigma}\left(g g \rightarrow \tilde{\chi}_{1}^{+} \tilde{\chi}_{1}^{-}, \quad \text { at } \hat{s}=\tau s\right) .
$$

where $\sqrt{s}$ and $\sqrt{\hat{s}}$ denote the proton-proton and gluon-gluon c.m.s. energies respectively 
and $\frac{d L_{g g}}{d \tau}$ is the gluon luminosity, which is defined as

$$
\frac{d \mathcal{L}_{g g}}{d \tau}=\int_{\tau}^{1} \frac{d x_{1}}{x_{1}}\left[F_{g}\left(x_{1}, \mu\right) F_{g}\left(\frac{\tau}{x_{1}}, \mu\right)\right] .
$$

Here we used $\tau=x_{1} x_{2}$, one can find the definitions of $x_{1}$ and $x_{2}$ in Ref. 22, and the energy scale $\mu$ is taken as $\mu=\sqrt{\hat{s}}$. We adopt the MRS set G parton distribution function $F_{g}(x)$ [23], and ignore the supersymmetric QCD corrections to the parton distribution functions for simplicity. The numerical calculation is carried out for the LHC at the energy around $10 \sim 14 T e V$.

The cross section for the process of the lightest higgsino-like chargino pair production $p p \rightarrow g g \rightarrow \tilde{\chi}_{1}^{+} \tilde{\chi}_{1}^{-}+X$ versus $\sqrt{s}$, with $m_{\tilde{\chi}_{1}^{+}}=165 \mathrm{GeV}, m_{\tilde{\chi}_{2}^{+}}=750 \mathrm{GeV}$, are depicted in Fig.6. The full and dashed lines are for $\tan \beta=4$ and 40 respectively, with all $\mathrm{CP}$ phase angles being zero. The dotted-line is for $\phi_{t}=\phi_{b}=\pi / 4$ and other CP phase angels being vanished. We can see that the total cross section at the future LHC collider can reach 56 femto barn for the higgsino-like chargino pair production, when $\sqrt{s} \sim 14 T e V$, $\tan \beta=40$ and all CP phase angles vanish. Calculating with the analytical expressions given in Ref. [27], the results show that with the same input parameters, when $\sqrt{s} \sim 14 T e V$ and $\tan \beta=40$, the cross section of the lightest chargino pair production via quark-antiquark annihilation can reach 317 femto barn for higgsino-like case in the CP-conserving MSSM theory. Therefore we can conclude that the chargino pair production via gluon-gluon fusion is competitive with the standard Drell-Yan production at the LHC and can be considered as a part of the NLO QCD correction to the Drell-Yan production process. In Fig.6 we can see that 
the production rate has the weak dependence on the c.m.s energy $\sqrt{s}$ for $\tan \beta=4$, but is strongly related to $\sqrt{s}$ for $\tan \beta=40$. The CP-violating effect in total cross section of the lightest chargino pair production in the LHC, is also obvious. The discrepancies between the total cross sections of the lightest chargino pair production predicted in the $\mathrm{CP}$-conserving and the CP-violating MSSM at the LHC, are about 20\% as shown in Fig.6.

\section{Summary}

In this paper, we studied the pair production process of the lightest chargino via gluongluon fusion at the LHC. The numerical analyse of its production rates is carried out in the MSSM with typical parameter sets. The results show that the cross section of the lightest chargino pair production via gluon-gluon fusion can be over 2.7 femto barn and the cross section at a future LHC collider can be 6.2 to 56 femto barn for the higgsino-like chargino pair production. It shows clearly that the production rates in proton-proton colliders can be largely enhanced if the chargino is higgsino-like. We find that the chargino production via gluon-gluon fusion could be competitive with the standard Drell-Yan production in the LHC and can be considered as a part of the NLO QCD correction to the Drell-Yan production subprocess. Our calculation shows also that in some exceptional c.m.s energy regions of incoming gluons, where the resonance conditions are satisfied in the parameter space, we can see observable enhancement effects on the curves. We also investigated the effects of complex phases $\phi_{q}$ in the squark mass matrices and $\phi_{\mu}$ appearing in chargino mass matrix in higgsino-like chargino case and found that the production rates in subprocess are sensitive to 
the CP-odd complex phases $\phi_{q}$ and $\phi_{\mu}$, but the effects from the phase angles $\phi_{q}$ are stronger than those from $\phi_{\mu}$. The effects from the CP-odd phase angles can be also demonstrated in the total cross section of the lightest chargino pair production in the LHC. Therefore it could be possible to get some information about these phase parameters, if we collected enough events statistically in searching for chargino pair via gluon-gluon fusion at the LHC.

Acknowledgement: These work was supported in part by the National Natural Science Foundation of China(project numbers: 19675033, 19875049), the Youth Science Foundation of the University of Science and Technology of China and a grant from the Education Ministry of China.

\section{Appendix}

A. The relevant Feynman rules of the MSSM.

The Feynman rules for the couplings of $q-\tilde{q}_{L, R}^{\prime}-\tilde{\chi}_{j=1,2}^{+}$are presented in Ref. [3] [4]. Then the corresponding Feynman rules for such vertices in squark mass eigenstate basis can be obtained as:

$$
\begin{array}{cc}
\bar{U}-\tilde{D}_{i}-\tilde{\chi}_{j}^{+}: & V_{U \tilde{D}_{i} \tilde{\chi}_{j}^{+}}^{(1)} P_{L}+V_{U \tilde{D}_{i} \tilde{\chi}_{j}^{+}}^{(2)} P_{R}, \\
U-\overline{\tilde{D}}_{i}-\overline{\tilde{\chi}}_{j}^{+}: & -V_{U \tilde{D}_{i} \tilde{\chi}_{j}^{+}}^{(2) *} P_{L}-V_{U \tilde{D}_{i} \tilde{\chi}_{j}^{+}}^{(1) *} P_{R}, \\
D-\overline{\tilde{U}}_{i}-\overline{\tilde{\chi}}_{j}^{+c}: \quad & C^{-1}\left\{V_{D \tilde{U}_{i} \tilde{\chi}_{j}^{+}}^{(1)} P_{L}+V_{D \tilde{U}_{i} \tilde{\chi}_{j}^{+}}^{(2)} P_{R}\right\}, \\
\bar{D}-\tilde{U}_{i}-\tilde{\chi}_{j}^{+c}: \quad & \left\{V_{D \tilde{U}_{i} \tilde{\chi}_{j}^{+}}^{(2) *} P_{L}+V_{D \tilde{U}_{i} \tilde{\chi}_{j}^{+}}^{(1) *} P_{R}\right\} C,
\end{array}
$$


respectively. Here $(U, D)=(u, d),(c, s),(t, b)$ and $\mathrm{C}$ is the charge conjugation matrix, which appears when there is a discontinuous flow of fermion number, $P_{L, R}=\frac{1}{2}\left(1 \mp \gamma_{5}\right)$ and

$$
\begin{gathered}
V_{U \tilde{D}_{1} \tilde{\chi}_{j}^{+}}^{(1)}=\frac{i g m_{U}}{\sqrt{2} m_{W} \sin \beta} V_{j 2}^{*} \cos \theta_{D} e^{-i \phi_{D}}, \\
V_{U \tilde{D}_{1} \tilde{\chi}_{j}^{+}}^{(2)}=-i g\left(U_{j 1} \cos \theta_{D} e^{-i \phi_{D}}+\frac{m_{D}}{\sqrt{2} m_{W} \cos \beta} U_{j 2} \sin \theta_{D} e^{i \phi_{D}}\right), \\
V_{U \tilde{D}_{2} \tilde{\chi}_{j}^{+}}^{(1)}=\frac{i g m_{U}}{\sqrt{2} m_{W} \sin \beta} V_{j 2}^{*} \sin \theta_{D} e^{-i \phi_{D}}, \\
V_{U \tilde{D}_{2} \tilde{\chi}_{j}^{+}}^{(2)}=-i g\left(U_{j 1} \sin \theta_{D} e^{-i \phi_{D}}-\frac{m_{D}}{\sqrt{2} m_{W} \cos \beta} U_{j 2} \cos \theta_{D} e^{i \phi_{D}}\right), \\
V_{D \tilde{U}_{1} \tilde{\chi}_{j}^{+}}^{(1)}=i g\left(V_{j 1}^{*} \cos \theta_{U} e^{i \phi_{U}}+\frac{m_{U}}{\sqrt{2} m_{W} \sin \beta} V_{j 2}^{*} \sin \theta_{U} e^{-i \phi_{D}}\right), \\
V_{D \tilde{U}_{1} \tilde{\chi}_{j}^{+}}^{(2)}=\frac{-i g m_{D}}{\sqrt{2} m_{W} \cos \beta} U_{j 2} \cos \theta_{U} e^{i \phi_{D}}, \\
V_{D \tilde{U}_{2} \tilde{\chi}_{j}^{+}}^{(1)}=i g\left(V_{j 1}^{*} \sin \theta_{U} e^{i \phi_{U}}-\frac{m_{U}}{\sqrt{2} m_{W} \sin \beta} V_{j 2}^{*} \cos \theta_{U} e^{-i \phi_{D}}\right), \\
V_{D \tilde{U}_{2} \tilde{\chi}_{j}^{+}}^{(2)}=\frac{i g m_{D}}{\sqrt{2} m_{W} \cos \beta} U_{i 2} \sin \theta_{U} e^{-i \phi_{D}},
\end{gathered}
$$

For the Feynman rules of the Higgs-quark-quark, Higgs-squark-squark, Higgs-chargino-chargino and $\mathrm{Z}(\gamma)$-chargino-chargino, one can refer to Ref. [3] 回. The couplings of $\operatorname{Higgs}(B)-\tilde{\chi}_{k}^{+}-\tilde{\chi}_{k}^{+}$ are

$$
V_{B \tilde{\chi}_{k}^{+} \tilde{\chi}_{k}^{+}}=V_{B \tilde{\chi}_{k}^{+} \tilde{\chi}_{k}^{+}}+V_{B \tilde{\chi}_{k}^{+} \tilde{\chi}_{k}^{+}}^{p s} \gamma_{5} \quad\left(B=h^{0}, H^{0}, A^{0}, G^{0}\right)
$$

where the notations defined above, which are involved in our calculation, are explicitly expressed as below:

$$
V_{H^{0} \tilde{\chi}_{k}^{+} \tilde{\chi}_{k}^{+}}^{s}=\frac{-i g}{\sqrt{2}}\left[\cos \alpha \operatorname{Re}\left(V_{k, 1} U_{k, 2}\right)+\sin \alpha \operatorname{Re}\left(V_{k, 2} U_{k, 1}\right)\right]
$$




$$
\begin{aligned}
V_{h^{0} \tilde{\chi}_{k}^{+} \tilde{\chi}_{k}^{+}}^{s} & =\frac{i g}{\sqrt{2}}\left[\sin \alpha \operatorname{Re}\left(V_{k, 1} U_{k, 2}\right)-\cos \alpha \operatorname{Re}\left(V_{k, 2} U_{k, 1}\right)\right] \\
V_{A^{0} \tilde{\chi}_{k}^{+} \tilde{\chi}_{k}^{+}}^{p s} & =\frac{g}{\sqrt{2}}\left[\sin \beta \operatorname{Re}\left(V_{k, 1} U_{k, 2}\right)+\cos \beta \operatorname{Re}\left(V_{k, 2} U_{k, 1}\right)\right] \\
V_{G^{0} \tilde{\chi}_{k}^{+} \tilde{\chi}_{k}^{+}}^{p s} & =\frac{-g}{\sqrt{2}}\left[\cos \beta \operatorname{Re}\left(V_{k, 1} U_{k, 2}\right)-\sin \beta R e\left(V_{k, 2} U_{k, 1}\right)\right]
\end{aligned}
$$

We define the following notations in Higgs-quark-quark couplings:

$$
\begin{gathered}
H^{0}-U-U: \quad V_{H^{0} U U}=\frac{-i g m_{U} \sin \alpha}{2 m_{W} \sin \beta}, \quad H^{0}-D-D: \quad V_{H^{0} D D}=\frac{-i g m_{D} \cos \alpha}{2 m_{W} \cos \beta}, \quad(A .5 .1) \\
h^{0}-U-U: \quad V_{h^{0} U U}=\frac{-i g m_{U} \cos \alpha}{2 m_{W} \sin \beta}, \quad h^{0}-D-D: \quad V_{h^{0} D D}=\frac{i g m_{D} \sin \alpha}{2 m_{W} \cos \delta}, \quad(A .5 .2) \\
A^{0}-U-U: \quad V_{A^{0} U U} \gamma_{5}=\frac{-g m_{U} \cot \beta}{2 m_{W}} \gamma_{5}, \quad A^{0}-D-D: \quad V_{A^{0} D D} \gamma_{5}=\frac{-g m_{D} \tan \beta}{2 m_{W}} \gamma_{5}, \\
G^{0}-U-U: \quad V_{G^{0} U U} \gamma_{5}=\frac{-g m_{U}}{2 m_{W}} \gamma_{5}, \quad G^{0}-D-D: \quad V_{G^{0} D D} \gamma_{5}=\frac{g m_{D}}{2 m_{W}} \gamma_{5} .
\end{gathered}
$$

The couplings of $H^{0}\left(h^{0}\right)-\tilde{q}_{i}-\tilde{q}_{i} \quad(i=1,2, q=u, d, c, s, t, b)$ are

$$
\begin{aligned}
V_{H^{0} \tilde{U}_{1} \tilde{U}_{1}}= & \frac{-i g m_{Z} \cos (\alpha+\beta)}{\cos \theta_{W}}\left[\left(\frac{1}{2}-\frac{2}{3} \sin ^{2} \theta_{W}\right) \cos ^{2} \theta_{U}+\frac{2}{3} \sin ^{2} \theta_{W} \sin ^{2} \theta_{U}\right] \\
& -\frac{i g m_{U}^{2} \sin \alpha}{m_{W} \sin \beta}+\frac{i g m_{U}}{2 m_{W} \sin \beta}\left(A_{U} \sin \alpha+\mu \cos \alpha\right) \sin \theta_{U} \cos \theta_{U} \cos 2 \phi_{U}, \\
V_{H^{0} \tilde{U}_{2} \tilde{U}_{2}}= & \frac{-i g m_{Z} \cos (\alpha+\beta)}{\cos \theta_{W}}\left[\left(\frac{1}{2}-\frac{2}{3} \sin ^{2} \theta_{W}\right) \sin ^{2} \theta_{U}+\frac{2}{3} \sin ^{2} \theta_{W} \cos ^{2} \theta_{U}\right] \\
& -\frac{i g m_{U}^{2} \sin \alpha}{m_{W} \sin \beta}-\frac{i g m_{U}}{2 m_{W} \sin \beta}\left(A_{U} \sin \alpha+\mu \cos \alpha\right) \sin \theta_{U} \cos \theta_{U} \cos 2 \phi_{U}, \\
V_{H^{0} \tilde{D}_{1} \tilde{D}_{1}}= & \frac{i g m_{Z} \cos (\alpha+\beta)}{\cos \theta_{W}}\left[\left(\frac{1}{2}-\frac{1}{3} \sin ^{2} \theta_{W}\right) \cos ^{2} \theta_{D}+\frac{1}{3} \sin { }^{2} \theta_{W} \sin ^{2} \theta_{D}\right] \\
& -\frac{i g m_{D}^{2} \cos \alpha}{m_{W} \cos \beta}+\frac{i g m_{D}}{2 m_{W} \cos \beta}\left(A_{D} \cos \alpha+\mu \sin \alpha\right) \sin \theta_{D} \cos \theta_{D} \cos 2 \phi_{D},
\end{aligned}
$$




$$
\begin{aligned}
& V_{H^{0} \tilde{D}_{2} \tilde{D}_{2}}=\frac{i g m_{Z} \cos (\alpha+\beta)}{\cos \theta_{W}}\left[\left(\frac{1}{2}-\frac{1}{3} \sin ^{2} \theta_{W}\right) \sin ^{2} \theta_{D}+\frac{1}{3} \sin ^{2} \theta_{W} \cos ^{2} \theta_{D}\right] \\
& -\frac{i g m_{D}^{2} \cos \alpha}{m_{W} \cos \beta}-\frac{i g m_{D}}{2 m_{W} \cos \beta}\left(A_{D} \cos \alpha+\mu \sin \alpha\right) \sin \theta_{D} \cos \theta_{D} \cos 2 \phi_{D}, \\
& V_{h^{0} \tilde{U}_{1} \tilde{U}_{1}}=\frac{i g m_{Z} \sin (\alpha+\beta)}{\cos \theta_{W}}\left[\left(\frac{1}{2}-\frac{2}{3} \sin ^{2} \theta_{W}\right) \cos ^{2} \theta_{U}+\frac{2}{3} \sin ^{2} \theta_{W} \sin ^{2} \theta_{U}\right] \\
& -\frac{i g m_{U}^{2} \cos \alpha}{m_{W} \sin \beta}+\frac{i g m_{U}}{2 m_{W} \sin \beta}\left(A_{U} \cos \alpha-\mu \sin \alpha\right) \sin \theta_{U} \cos \theta_{U} \cos 2 \phi_{U}, \\
& V_{h^{0} \tilde{U}_{2} \tilde{U}_{2}}=\frac{i g m_{Z} \sin (\alpha+\beta)}{\cos \theta_{W}}\left[\left(\frac{1}{2}-\frac{2}{3} \sin ^{2} \theta_{W}\right) \sin ^{2} \theta_{U}+\frac{2}{3} \sin ^{2} \theta_{W} \cos ^{2} \theta_{U}\right] \\
& -\frac{i g m_{U}^{2} \cos \alpha}{m_{W} \sin \beta}-\frac{i g m_{U}}{2 m_{W} \sin \beta}\left(A_{U} \cos \alpha-\mu \sin \alpha\right) \sin \theta_{U} \cos \theta_{U} \cos 2 \phi_{U}, \\
& V_{h^{0} \tilde{D}_{1} \tilde{D}_{1}}=\frac{-i g m_{Z} \sin (\alpha+\beta)}{\cos \theta_{W}}\left[\left(\frac{1}{2}-\frac{1}{3} \sin ^{2} \theta_{W}\right) \cos ^{2} \theta_{D}+\frac{1}{3} \sin ^{2} \theta_{W} \sin ^{2} \theta_{D}\right] \\
& +\frac{i g m_{D}^{2} \sin \alpha}{m_{W} \cos \beta}-\frac{i g m_{D}}{2 m_{W} \cos \beta}\left(A_{D} \sin \alpha-\mu \cos \alpha\right) \sin \theta_{D} \cos \theta_{D} \cos 2 \phi_{D}, \\
& V_{h^{0} \tilde{D}_{2} \tilde{D}_{2}}=\frac{-i g m_{Z} \sin (\alpha+\beta)}{\cos \theta_{W}}\left[\left(\frac{1}{2}-\frac{1}{3} \sin ^{2} \theta_{W}\right) \sin ^{2} \theta_{D}+\frac{1}{3} \sin ^{2} \theta_{W} \cos ^{2} \theta_{D}\right] \\
& +\frac{i g m_{D}^{2} \sin \alpha}{m_{W} \cos \beta}+\frac{i g m_{D}}{2 m_{W} \cos \beta}\left(A_{D} \sin \alpha-\mu \cos \alpha\right) \sin \theta_{D} \cos \theta_{D} \cos 2 \phi_{D},
\end{aligned}
$$

respectively.

\section{B. Form Factors.}

As mentioned above, the amplitude parts from the u-channel box and triangle vertex interaction diagrams can be obtained from the t-channel's, so we present only the t-channel form factors for box and triangle diagrams. Since the form factors of the first and second generation (s)quarks are analogous to those of the third generation (s)quarks, here we list 
only the form factors of the box, triangle and quartic interaction parts for the third generation quarks and squarks. Actually we should take the sum of the form factors of each generation (s)quarks for the total form factors. In appendix, we use the notations defined in below for abbreviation.

$$
\begin{gathered}
\bar{B}_{0}^{1, k}=B_{0}\left[-p_{1}-p_{2}, m_{\tilde{t}_{k}}, m_{\tilde{t}_{k}}\right]-\Delta, \quad \bar{B}_{0}^{2, k}=B_{0}\left[-p_{1}-p_{2}, m_{\tilde{b}_{k}}, m_{\tilde{b}_{k}}\right]-\Delta \\
C_{0}^{1}, C_{i j}^{1}=C_{0}, C_{i j}\left[p_{3},-p_{1}-p_{2}, m_{t}, m_{t}, m_{t}\right] \\
C_{0}^{2}, C_{i j}^{2}=C_{0}, C_{i j}\left[p_{3},-p_{1}-p_{2}, m_{b}, m_{b}, m_{b}\right] \\
C_{0}^{3, k}, C_{i j}^{3, k}=C_{0}, C_{i j}\left[p_{3},-p_{1}-p_{2}, m_{\tilde{t}_{k}}, m_{\tilde{t}_{k}}, m_{\tilde{t}_{k}}\right] \\
C_{0}^{4, k}, C_{i j}^{4, k}=C_{0}, C_{i j}\left[p_{3},-p_{1}-p_{2}, m_{\tilde{b}_{k}}, m_{\tilde{b}_{k}}, m_{\tilde{b}_{k}}\right] \\
C_{0}^{5, k}, C_{i j}^{5, k}=C_{0}, C_{i j}\left[-p_{1}, p_{1}+p_{2}, m_{t}, m_{\tilde{b}_{k}}, m_{\tilde{b}_{k}}\right] \\
C_{0}^{6, k}, C_{i j}^{6, k}=C_{0}, C_{i j}\left[-p_{1}, p_{1}+p_{2}, m_{b}, m_{\tilde{t}_{k}}, m_{\tilde{t}_{k}}\right] \\
D_{0}^{1, k}, D_{i j}^{1, k}, D_{i j l}^{1, k}=D_{0}, D_{i j}, D_{i j l}\left[-p_{1}, p_{3}, p_{4}, m_{\tilde{b}_{k}}, m_{t}, m_{t}, m_{t}\right] \\
D_{0}^{2, k}, D_{i j}^{2, k}, D_{i j l}^{2, k}=D_{0}, D_{i j}, D_{i j l}\left[-p_{1}, p_{3}, p_{4}, m_{\tilde{t}_{k}}, m_{b}, m_{b}, m_{b}\right] \\
D_{0}^{3, k}, D_{i j}^{3, k}, D_{i j l}^{3, k}=D_{0}, D_{i j}, D_{i j l}\left[-p_{1}, p_{3}, p_{4}, m_{t}, m_{\tilde{b}_{k}}, m_{\tilde{b}_{k}}, m_{\tilde{b}_{k}}\right] \\
D_{0}^{4, k}, D_{i j}^{4, k}, D_{i j l}^{4, k}=D_{0}, D_{i j}, D_{i j l}\left[-p_{1}, p_{3}, p_{4}, m_{b}, m_{\tilde{t}_{k}}, m_{\tilde{t}_{k}}, m_{\tilde{t}_{k}}\right] \\
D_{0}^{5, k}, D_{i j}^{5, k}, D_{i j l}^{5, k}=D_{i j l}^{6, k}=D_{0}, D_{i j}, D_{i j l}\left[p_{3}, p_{2}-p_{3},-p_{4}, m_{t}, m_{t}, m_{\tilde{b}_{k}}, m_{\tilde{b}_{k}}\right] \\
\left.D_{3 j}, p_{2}-p_{3},-p_{4}, m_{b}, m_{b}, m_{\tilde{t}_{k}}, m_{\tilde{t}_{k}}\right]
\end{gathered}
$$




$$
\begin{aligned}
& A_{t}=\frac{i}{\hat{t}-m_{\tilde{\chi}_{1}^{+}}^{2}}, \quad A_{u}=\frac{i}{\hat{u}-m_{\tilde{\chi}_{1}^{+}}^{2}} \\
& A_{h}=\frac{i}{\hat{s}-m_{h}^{2}}, \quad A_{H}=\frac{i}{\hat{s}-m_{H}^{2}} . \\
& A_{A}=\frac{i}{\hat{s}-m_{A}^{2}}, \quad A_{G}=\frac{i}{\hat{s}-m_{Z}^{2}} \\
& F_{1, k}=-V_{t \tilde{b}_{k} \tilde{\chi}_{1}^{+}}^{(1)} V_{t \tilde{b}_{k} \tilde{\chi}_{1}^{+}}^{(2) *}-V_{t \tilde{b}_{k} \tilde{\chi}_{1}^{+}}^{(2)} V_{t \tilde{b}_{k} \tilde{\chi}_{1}^{+}}^{(1) *}, \quad F_{2, k}=-\left|V_{t \tilde{b}_{k} \tilde{\chi}_{1}^{+}}^{(1)}\right|^{2}-\left|V_{t \tilde{b}_{k} \tilde{\chi}_{1}^{+}}^{(2)}\right|^{2} \\
& F_{3, k}=-V_{t \tilde{b}_{k} \tilde{\chi}_{1}^{+}}^{(1)} V_{t \tilde{b}_{k} \tilde{\chi}_{1}^{+}}^{(2) *}+V_{t \tilde{b}_{k} \tilde{\chi}_{1}^{+}}^{(2)} V_{t \tilde{b}_{k} \tilde{\chi}_{1}^{+}}^{(1) *}, \quad F_{4, k}=-\left|V_{t \tilde{b}_{k} \tilde{\chi}_{1}^{+}}^{(1)}\right|^{2}+\left|V_{t \tilde{b}_{k} \tilde{\chi}_{1}^{+}}^{(2)}\right|^{2} \\
& F_{5, k}=V_{b \tilde{t}_{k} \tilde{\chi}_{1}^{+}}^{(1)} V_{b \tilde{t}_{k} \tilde{\chi}_{1}^{+}}^{(2) *}+V_{b \tilde{t}_{k} \tilde{\chi}_{1}^{+}}^{(2)} V_{b \tilde{t}_{k} \tilde{\chi}_{1}^{+}}^{(1) *}, \quad F_{6, k}=\left|V_{b \tilde{t}_{k} \tilde{\chi}_{1}^{+}}^{(1)}\right|^{2}+\left|V_{b \tilde{t}_{k} \tilde{\chi}_{1}^{+}}^{(2)}\right|^{2} \\
& F_{7, k}=V_{b \tilde{t}_{k} \tilde{\chi}_{1}^{+}}^{(1)} V_{b \tilde{t}_{k} \tilde{\chi}_{1}^{+}}^{(2) *}-V_{b \tilde{t}_{k} \tilde{\chi}_{1}^{+}}^{(2)} V_{b \tilde{t}_{k} \tilde{\chi}_{1}^{+}}^{(1) *}, \quad F_{8, k}=\left|V_{b \tilde{t}_{k} \tilde{\chi}_{1}^{+}}^{(1)}\right|^{2}-\left|V_{b \tilde{t}_{k} \tilde{\chi}_{1}^{+}}^{(2)}\right|^{2}
\end{aligned}
$$

In the following, the expression denoted as $(t \rightarrow b)$ means doing the replacements of $Q_{t} \rightarrow Q_{b}$, $m_{t} \rightarrow m_{b}, F_{1, k} \rightarrow F_{5, k}, F_{2, k} \rightarrow F_{6, k}, F_{3, k} \rightarrow F_{7, k}, F_{4, k} \rightarrow F_{8, k}, B_{0}^{1, k} \rightarrow B_{0}^{2, k}, C^{1} \rightarrow C^{2}$, $C^{3, k} \rightarrow C^{4, k}, C^{5, k} \rightarrow C^{6, k}, D^{1, k} \rightarrow D^{2, k}, D^{3, k} \rightarrow D^{4, k}, D^{5, k} \rightarrow D^{6, k}$. The form factors in the amplitude of the quartic interaction diagrams Fig.1(b) are expressed as:

$$
\begin{aligned}
& f_{1}^{q}=f_{2}^{q}= \frac{g_{s}^{2}}{32 \pi^{2}} \sum_{k=1,2}\left[2 \bar{B}_{0}^{1, k}\left(A_{h} V_{h^{0} \tilde{t}_{k} \tilde{t}_{k}} V_{h^{0} \tilde{\chi}_{1}^{+} \tilde{\chi}_{1}^{+}}^{s}+A_{H} V_{H^{0} \tilde{t}_{k} \tilde{t}_{k}} V_{H^{0} \tilde{\chi}_{1}^{+} \tilde{\chi}_{1}^{+}}^{s}\right)\right. \\
&-\left.i\left(C_{0}^{5, k} m_{t} F_{1, k}-C_{11}^{5, k} m_{\tilde{\chi}_{1}^{+}} F_{2, k}\right)\right]+(t \rightarrow b) \\
& f_{34}^{q}=f_{35}^{q}= \frac{g_{s}^{2}}{32 \pi^{2}} \sum_{k=1,2}\left\{2 \bar{B}_{0}^{1, k}\left(A_{h} V_{h^{0} \tilde{t}_{k} \tilde{t}_{k}} V_{h^{0} \tilde{\chi}_{1}^{+} \tilde{\chi}_{1}^{+}}^{p s}+A_{H} V_{H^{0} \tilde{t}_{k} \tilde{t}_{k}} V_{H^{0} \tilde{\chi}_{1}^{+} \tilde{\chi}_{1}^{+}}^{p s}\right)\right. \\
&+\left.i\left[C_{0}^{5, k} m_{t} F_{3, k}+\left(2 C_{12}^{5, k}-C_{11}^{5, k}\right) m_{\tilde{\chi}_{1}^{+}} F_{4, k}\right]\right\}+(t \rightarrow b) \\
& f_{i}^{q}=0 . \quad(i=3 \sim 33,36 \sim 66)
\end{aligned}
$$


The form factors in the amplitude from the t-channel triangle diagrams depicted in Fig.1(c), are listed below:

$$
\begin{aligned}
& f_{1}^{t r, \hat{t}}=f_{2}^{t r, \hat{t}} \\
& =\frac{g_{s}^{2}}{8 \pi^{2}}\left\{m _ { t } \left(A_{h} V_{h^{0} t t} V_{h^{0} \tilde{\chi}_{1}^{+} \tilde{\chi}_{1}^{+}}^{s}+A_{H} V_{H^{0} t t} V_{H^{0} \tilde{\chi}_{1}^{+} \tilde{\chi}_{1}^{+}}^{s}\left[-C_{0}^{1} m_{t}^{2}+2 C_{22}^{1}\left(p_{1} \cdot p_{2}+m_{\tilde{\chi}_{1}^{+}}^{2}\right)\right.\right.\right. \\
& \left.\left.+\left(C_{0}^{1}-2 C_{23}^{1}\right)\left(p_{1}+p_{2}\right) \cdot p_{3}\right]-\sum_{k=1,2} 2 \bar{C}_{24}^{3, k}\left(A_{h} V_{h^{0} \tilde{t}_{k} \tilde{t}_{k}} V_{h^{0} \tilde{\chi}_{1}^{+} \tilde{\chi}_{1}^{+}}^{s}+A_{H} V_{H^{0} \tilde{t}_{k} \tilde{t}_{k}} V_{H^{0} \tilde{\chi}_{1}^{+} \tilde{\chi}_{1}^{+}}^{s}\right)\right\} \\
& +(t \rightarrow b) \\
& f_{3}^{t r, \hat{t}}=f_{4}^{t r, \hat{t}}=\frac{g_{s}^{2}}{4 \pi^{2}}\left(\frac{i e^{2} Q_{t}}{\hat{s}}-A_{G} V_{Z^{0} t t}^{v} V_{Z^{0} \tilde{\chi}_{1}^{+} \tilde{\chi}_{1}^{+}}^{+}\right)\left[2\left(\bar{C}_{24}^{1}+\bar{C}_{36}^{1}-\bar{C}_{35}^{1}\right)\right. \\
& +\left(C_{0}^{1}+C_{12}^{1}-C_{11}^{1}\right) m_{t}^{2}+2\left(C_{34}^{1}-C_{12}^{1}-C_{22}^{1}-C_{32}^{1}\right)\left(p_{1} \cdot p_{2}+m_{\tilde{\chi}_{1}^{+}}^{2}\right) \\
& \left.+2\left(C_{12}^{1}+C_{22}^{1}+C_{34}^{1}-C_{33}^{1}\right)\left(p_{1}+p_{2}\right) \cdot p_{3}\right]+(t \rightarrow b) \\
& f_{5}^{t r, \hat{t}}=f_{6}^{t r, \hat{t}}=-\frac{g_{s}^{2}}{4 \pi^{2}}\left(\frac{i e^{2} Q_{t}}{\hat{s}}-A_{G} V_{Z^{0} t t}^{v} V_{Z^{0}}^{s} \tilde{\chi}_{1}^{+} \tilde{\chi}_{1}^{+}\right)\left[2 \bar{C}_{24}^{1}-2 \bar{C}_{36}^{1}+\left(C_{0}^{1}-C_{12}^{1}\right) m_{t}^{2}\right. \\
& \left.+2\left(C_{22}^{1}+C_{32}^{1}\right)\left(p_{1} \cdot p_{2}+m_{\tilde{\chi}_{1}^{+}}^{2}\right)-2\left(C_{22}^{1}+C_{34}^{1}\right)\left(p_{1}+p_{2}\right) \cdot p_{3}\right]+(t \rightarrow b) \\
& f_{7}^{t r, \hat{t}}=f_{8}^{t r, \hat{t}}=f_{9}^{t r, \hat{t}}=f_{10}^{t r, \hat{t}}=\frac{g_{s}^{2}}{4 \pi^{2}}\left[m_{t}\left(A_{h} V_{h^{0} t t} V_{h^{0} \tilde{\chi}_{1}^{+} \tilde{\chi}_{1}^{+}}+A_{H} V_{H^{0} t t} V_{H^{0} \tilde{\chi}_{1}^{+} \tilde{\chi}_{1}^{+}}^{s}\right)\left(4 C_{23}^{1}-C_{0}^{1}-4 C_{22}^{1}\right)\right. \\
& \left.+\sum_{k=1,2} 2\left(A_{h} V_{h^{0} \tilde{t}_{k} \tilde{t}_{k}} V_{h^{0} \tilde{\chi}_{1}^{+} \tilde{\chi}_{1}^{+}}^{s}+A_{H} V_{H^{0} \tilde{t}_{k} t \tilde{t}_{k}} V_{H^{0} \tilde{\chi}_{1}^{+} \tilde{\chi}_{1}^{+}}^{s}\right)\left(C_{22}^{3, k}-C_{23}^{3, k}\right)\right]+(t \rightarrow b) \\
& f_{11}^{t r, \hat{t}}=f_{12}^{t r, \hat{t}}=\frac{g_{s}^{2}}{8 \pi^{2}}\left(\frac{i e^{2} Q_{t}}{\hat{s}}-A_{G} V_{Z^{0} t t}^{v} V_{Z^{0}}^{s} \tilde{\chi}_{1}^{+} \tilde{\chi}_{1}^{+}\right)\left[-4 \bar{C}_{24}^{1}-2 \bar{C}_{35}^{1}\right. \\
& -\left(2 C_{0}^{1}+C_{11}^{1}\right) m_{t}^{2}+2\left(C_{12}^{1}+C_{34}^{1}+2 C_{22}^{1}\right)\left(p_{1} \cdot p_{2}+m_{\tilde{\chi}_{1}^{+}}^{2}\right) \\
& \left.-2\left(C_{12}^{1}+C_{33}^{1}+2 C_{23}^{1}\right)\left(p_{1}+p_{2}\right) \cdot p_{3}\right]+(t \rightarrow b)
\end{aligned}
$$




$$
\begin{aligned}
& f_{17}^{t r, \hat{t}}=f_{18}^{t r, \hat{t}}=f_{19}^{t r, \hat{t}}=f_{20}^{t r, \hat{t}}=-\frac{g_{s}^{2}}{\pi^{2}}\left(\frac{i e^{2} Q_{t}}{4 \hat{s}}-A_{G} V_{Z^{0} t t}^{v} V_{Z^{0}}^{s} \tilde{\chi}_{1}^{+} \tilde{\chi}_{1}^{+}\right)\left(C_{22}^{1}+C_{34}^{1}-C_{23}^{1}-C_{33}^{1}\right) \\
& +(t \rightarrow b) \\
& f_{21}^{t r, \hat{t}}=f_{22}^{t r, \hat{t}}=-\frac{i g_{s}^{2} m_{t}}{4 \pi^{2}} C_{0}^{1}\left(A_{A} V_{A^{0} t t} V_{A^{0} \tilde{\chi}_{1}^{+} \tilde{\chi}_{1}^{+}}+A_{G} V_{G^{0} t t} V_{G^{0}}^{s} \tilde{\chi}_{1}^{+} \tilde{\chi}_{1}^{+}\right)+(t \rightarrow b) \\
& f_{23}^{t r, \hat{t}}=f_{24}^{t r, \hat{t}}=\frac{i g_{s}^{2}}{4 \pi^{2}} A_{G} V_{Z^{0} t t}^{p v} V_{Z^{0} \tilde{\chi}_{1}^{+} \tilde{\chi}_{1}^{+}}^{s}-2 \bar{C}_{24}^{1}-6 \bar{C}_{36}^{1}-\left(C_{0}^{1}+C_{12}^{1}\right) m_{t}^{2} \\
& \left.+2\left(C_{22}^{1}+C_{32}^{1}\right)\left(p_{1} \cdot p_{2}+m_{\tilde{\chi}_{1}^{+}}^{2}\right)-2\left(C_{23}^{1}+C_{34}^{1}\right)\left(p_{1}+p_{2}\right) \cdot p_{3}\right]+(t \rightarrow b) \\
& f_{25}^{t r, \hat{t}}=-\frac{i g_{s}^{2}}{4 \pi^{2}} A_{G} V_{Z^{0} t t}^{p v} V_{Z^{0}}^{s} \tilde{\chi}_{1}^{+} \tilde{\chi}_{1}^{+}\left[-4 \bar{C}_{24}^{1}-6 \bar{C}_{35}^{1}-\left(2 C_{0}^{1}+C_{11}^{1}\right) m_{t}^{2}\right. \\
& \left.+2\left(C_{12}^{1}+C_{34}^{1}+2 C_{22}^{1}\right)\left(p_{1} \cdot p_{2}+m_{\tilde{\chi}_{1}^{+}}^{2}\right)-2\left(C_{12}^{1}+C_{33}^{1}+2 C_{23}^{1}\right)\left(p_{1}+p_{2}\right) \cdot p_{3}\right] \\
& +(t \rightarrow b) \\
& f_{26}^{t r, \hat{t}}=f_{27}^{t r, \hat{t}}=f_{28}^{t r, \hat{t}}=f_{29}^{t r, \hat{t}}=\frac{i g_{s}^{2}}{2 \pi^{2}} A_{G} V_{Z^{0} t t}^{p v} V_{Z^{0}}^{s} \tilde{\chi}_{1}^{+} \tilde{\chi}_{1}^{+}\left(C_{22}^{1}-C_{23}^{1}\right)+(t \rightarrow b) \\
& f_{30}^{t r, \hat{t}}=f_{31}^{t r, \hat{t}}=f_{32}^{t r, \hat{t}}=f_{33}^{t r, \hat{t}}=-f_{26}^{t r, \hat{t}} \\
& f_{34}^{t r, \hat{t}}=f_{35}^{t r, \hat{t}}=-\frac{g_{s}^{2}}{4 \pi^{2}}\left\{\sum_{k=1,2} \bar{C}_{24}^{3, k}\left(A_{h} V_{h^{0} \tilde{t}_{k} \tilde{t}_{k}} V_{h^{0} \tilde{\chi}_{1}^{+} \tilde{\chi}_{1}^{+}}^{p s}+A_{H} V_{H^{0} \tilde{t}_{k} t \tilde{t}_{k}} V_{H^{0} \tilde{\chi}_{1}^{+} \tilde{\chi}_{1}^{+}}^{p s}\right)\right. \\
& +A_{G} V_{Z^{0} t t}^{v} V_{Z^{0} \tilde{\chi}_{1}^{+} \tilde{\chi}_{1}^{+}}^{p s}\left[2 \bar{C}_{24}^{1}+2 \bar{C}_{36}^{1}+\left(C_{0}^{1}+C_{12}^{1}\right) m_{t}^{2}\right. \\
& \left.\left.-2\left(C_{22}^{1}+C_{32}^{1}\right)\left(p_{1} \cdot p_{2}+m_{\tilde{\chi}_{1}^{+}}^{2}\right)+2\left(C_{23}^{1}+C_{34}^{1}\right)\left(p_{1}+p_{2}\right) \cdot p_{3}\right]\right\}+(t \rightarrow b) \\
& f_{36}^{t r, \hat{t}}=f_{37}^{t r, \hat{t}}=\frac{g_{s}^{2}}{4 \pi^{2}} A_{G} V_{Z^{0} t t}^{v} V_{Z^{0} \tilde{\chi}_{1}^{+} \tilde{\chi}_{1}^{+}}^{p s}\left[2 \bar{C}_{24}^{1}+2 \bar{C}_{36}^{1}-2 \bar{C}_{35}^{1}\right. \\
& +\left(C_{0}^{1}+C_{12}^{1}-C_{11}^{1}\right) m_{t}^{2}+2\left(C_{34}^{1}-C_{12}^{1}-C_{22}^{1}-C_{32}^{1}\right)\left(p_{1} \cdot p_{2}+m_{\tilde{\chi}_{1}^{+}}^{2}\right) \\
& \left.+2\left(C_{12}^{1}+C_{22}^{1}+C_{34}^{1}-C_{33}^{1}\right)\left(p_{1}+p_{2}\right) \cdot p_{3}\right]+(t \rightarrow b)
\end{aligned}
$$




$$
\begin{aligned}
& f_{38}^{t r, \hat{t}}=f_{39}^{t r, \hat{t}}=-\frac{g_{s}^{2}}{4 \pi^{2}} A_{G} V_{Z^{0} t t}^{v} V_{Z^{0} \tilde{\chi}_{1}^{+} \tilde{\chi}_{1}^{+}}^{p s}\left[2 \bar{C}_{24}^{1}-2 \bar{C}_{36}^{1}+\left(C_{0}^{1}-C_{12}^{1}\right) m_{t}^{2}\right. \\
& \left.+2\left(C_{22}^{1}+C_{32}^{1}\right)\left(p_{1} \cdot p_{2}+m_{\tilde{\chi}_{1}^{+}}^{2}\right)-2\left(C_{22}^{1}+C_{34}^{1}\right)\left(p_{1}+p_{2}\right) \cdot p_{3}\right]+(t \rightarrow b) \\
& f_{40}^{t r, \hat{t}}=f_{41}^{t r, \hat{t}}=f_{42}^{t r, \hat{t}}=f_{43}^{t r, \hat{t}}=\frac{g_{s}^{2}}{4 \pi^{2}}\left\{m_{t}\left(A_{h} V_{h^{0} t t} V_{h^{0} \tilde{\chi}_{1}^{+} \tilde{\chi}_{1}^{+}}^{p s}+A_{H} V_{H^{0} t t} V_{H^{0} \tilde{\chi}_{1}^{+} \tilde{\chi}_{1}^{+}}^{p s}\right)\left(4 C_{23}^{1}-C_{0}^{1}-4 C_{22}^{1}\right)\right. \\
& +4 A_{G} V_{Z^{0} t t}^{v} V_{Z^{0} \tilde{\chi}_{1}^{+} \tilde{\chi}_{1}^{+}}^{p s}\left(C_{23}^{1}+2 C_{34}^{1}-C_{22}^{1}-2 C_{32}^{1}\right) \\
& +\sum_{k=1,2} 2\left(C_{22}^{3, k}-C_{23}^{3, k}\right)\left(A_{h} V_{h^{0} \tilde{t}_{k} \tilde{t}_{k}} V_{h^{0} \tilde{\chi}_{1}^{+} \tilde{\chi}_{1}^{+}}^{p s}+A_{H} V_{H^{0} \tilde{t}_{k} t \tilde{t}_{k}} V_{H^{0} \tilde{\chi}_{1}^{+} \tilde{\chi}_{1}^{+}}^{p s}\right)+(t \rightarrow b) \\
& f_{44}^{t r, \hat{t}}=f_{45}^{t r, \hat{t}}=\frac{g_{s}^{2}}{8 \pi^{2}} A_{G} V_{Z^{0} t t}^{v} V_{Z^{0} \tilde{\chi}_{1}^{+} \tilde{\chi}_{1}^{+}}^{p s}\left[-4 \bar{C}_{24}^{1}-2 \bar{C}_{35}^{1}-\left(2 C_{0}^{1}+C_{11}^{1}\right) m_{t}^{2}\right. \\
& \left.+2\left(C_{12}^{1}+C_{34}^{1}+2 C_{22}^{1}\right)\left(p_{1} \cdot p_{2}+m_{\tilde{\chi}_{1}^{+}}^{2}\right)-2\left(C_{12}^{1}+C_{33}^{1}+2 C_{23}^{1}\right)\left(p_{1}+p_{2}\right) \cdot p_{3}\right] \\
& +(t \rightarrow b) \\
& f_{50}^{t r, \hat{t}}=f_{51}^{t r, \hat{t}}=f_{52}^{t r, \hat{t}}=f_{53}^{t r, \hat{t}}=-\frac{g_{s}^{2}}{\pi^{2}} A_{G} V_{Z^{0} t t}^{v} V_{Z^{0} \tilde{\chi}_{1}^{+} \tilde{\chi}_{1}^{+}}^{p s}\left(C_{22}^{1}+C_{34}^{1}-C_{23}^{1}-C_{33}^{1}\right)+(t \rightarrow b) \\
& f_{54}^{t r, \hat{t}}=f_{55}^{t r, \hat{t}}=-\frac{i g_{s}^{2} m_{t}}{4 \pi^{2}} C_{0}^{1}\left(A_{A} V_{A^{0} t t} V_{A^{0} \tilde{\chi}_{1}^{+} \tilde{\chi}_{1}^{+}}^{p s}+A_{G} V_{G^{0} t t} V_{G^{0} \tilde{\chi}_{1}^{+} \tilde{\chi}_{1}^{+}}^{p s}+(t \rightarrow b)\right. \\
& f_{56}^{t r, \hat{t}}=f_{57}^{t r, \hat{t}}=-\frac{i g_{s}^{2}}{4 \pi^{2}} A_{G} V_{Z^{0} t t}^{p v} V_{Z^{0} \tilde{\chi}_{1}^{+} \tilde{\chi}_{1}^{+}}^{p s}\left[-2 \bar{C}_{24}^{1}-6 \bar{C}_{36}^{1}-\left(C_{0}^{1}+C_{12}^{1}\right) m_{t}^{2}\right. \\
& \left.+2\left(C_{22}^{1}+C_{32}^{1}\right)\left(p_{1} \cdot p_{2}+m_{\tilde{\chi}_{1}^{+}}^{2}\right)-2\left(C_{23}^{1}+C_{34}^{1}\right)\left(p_{1}+p_{2}\right) \cdot p_{3}\right]+(t \rightarrow b) \\
& f_{58}^{t r, \hat{t}}=\frac{i g_{s}^{2}}{4 \pi^{2}} A_{G} V_{Z^{0} t t}^{p v} V_{Z^{0} \tilde{\chi}_{1}^{+} \tilde{\chi}_{1}^{+}}^{p s}\left[-4 \bar{C}_{24}^{1}-6 \bar{C}_{35}^{1}-\left(2 C_{0}^{1}+C_{11}^{1}\right) m_{t}^{2}\right. \\
& \left.+2\left(C_{12}^{1}+2 C_{22}^{1}+C_{34}^{1}\right)\left(p_{1} \cdot p_{2}+m_{\tilde{\chi}_{1}^{+}}^{2}\right)-2\left(C_{12}^{1}+2 C_{23}^{1}+C_{33}^{1}\right)\left(p_{1}+p_{2}\right) \cdot p_{3}\right] \\
& +(t \rightarrow b)
\end{aligned}
$$




$$
\begin{aligned}
& f_{59}^{t r, \hat{t}}=f_{60}^{t r, \hat{t}}=f_{61}^{t r, \hat{t}}=f_{62}^{t r, \hat{t}}=\frac{i g_{s}^{2}}{2 \pi^{2}} A_{G} V_{Z^{0} t t}^{p v} V_{Z^{0} \tilde{\chi}_{1}^{+} \tilde{\chi}_{1}^{+}}^{p s}\left(C_{23}^{1}-C_{22}^{1}\right)+(t \rightarrow b) \\
& f_{63}^{t r, \hat{t}}=f_{64}^{t r, \hat{t}}=f_{65}^{t r, \hat{t}}=f_{66}^{t r, \hat{t}}=-f_{59}^{t r, \hat{t}} \\
& f_{i}^{t r, \hat{t}}=0 \quad(i=13 \sim 16,46 \sim 49)
\end{aligned}
$$

where $\bar{C}_{24}=C_{24}-\frac{\Delta}{4}, \bar{C}_{35}=C_{35}+\frac{\Delta}{6}$ and $\bar{C}_{36}=C_{36}+\frac{\Delta}{12}$. The form factors of the amplitude part from t-channel box diagrams Fig.1(a) are written as:

$$
\begin{aligned}
& f_{1}^{b, \hat{t}}=\frac{i g_{s}^{2}}{32 \pi^{2}} \sum_{k=1,2}\left\{m _ { t } F _ { 1 , k } \left[2\left(D_{23}^{1, k}-D_{13}^{1, k}-D_{25}^{1, k}\right) p_{1} \cdot p_{2}+2\left(D_{25}^{1, k}+D_{26}^{1, k}+2 D_{13}^{1, k}-D_{11}^{1, k}\right.\right.\right. \\
& \left.-D_{12}^{1, k}-D_{23}^{1, k}-D_{24}^{1, k}\right) p_{1} \cdot p_{3}+2\left(D_{13}^{1, k}+D_{26}^{1, k}-D_{23}^{1, k}\right) p_{2} \cdot p_{3}+\left(2 D_{11}^{1, k}+2 D_{23}^{1, k}\right. \\
& \left.\left.+D_{0}^{1, k}+D_{21}^{1, k}-2 D_{13}^{1, k}-2 D_{25}^{1, k}\right) m_{\tilde{\chi}_{1}^{+}}^{2}-4 D_{27}^{1, k}+2\left(D_{27}^{5, k}+D_{27}^{1, k}+D_{27}^{3, k}\right)-D_{0}^{1, k} m_{t}^{2}\right] \\
& +m_{\tilde{\chi}_{1}^{+}} F_{2, k}\left[2\left(D_{23}^{1, k}+D_{37}^{1, k}-D_{13}^{1, k}-D_{35}^{1, k}-2 D_{25}^{1, k}\right) p_{1} \cdot p_{2}+2\left(3 D_{25}^{1, k}+D_{26}^{1, k}+D_{310}^{1, k}\right.\right. \\
& \left.+D_{35}^{1, k}+2 D_{13}^{1, k}-D_{11}^{1, k}-D_{12}^{1, k}-D_{21}^{1, k}-D_{23}^{1, k}-D_{34}^{1, k}-D_{37}^{1, k}-2 D_{24}^{1, k}\right) p_{1} \cdot p_{3} \\
& +2\left(D_{13}^{1, k}+D_{25}^{1, k}+D_{26}^{1, k}+D_{310}^{1, k}-D_{23}^{1, k}-D_{37}^{1, k}\right) p_{2} \cdot p_{3}-\left(D_{0}^{1, k}+D_{11}^{1, k}\right) m_{t}^{2} \\
& -4\left(D_{27}^{1, k}+D_{311}^{1, k}\right)-2\left(D_{312}^{5, k}+D_{311}^{3, k}\right) \\
& \left.\left.+\left(3 D_{11}^{1, k}+3 D_{21}^{1, k}+2 D_{23}^{1, k}+2 D_{37}^{1, k}+D_{0}^{1, k}+D_{31}^{1, k}-2 D_{13}^{1, k}-2 D_{35}^{1, k}-4 D_{25}^{1, k}\right) m_{\tilde{\chi}_{1}^{+}}^{2}\right]\right\} \\
& +(t \rightarrow b) \\
& f_{2}^{b, \hat{t}}=\frac{i g_{s}^{2}}{16 \pi^{2}} \sum_{k=1,2}\left[m_{t} F_{1, k}\left(D_{27}^{1, k}+D_{27}^{3, k}+D_{27}^{5, k}\right)+m_{\tilde{\chi}_{1}^{+}} F_{2, k}\left(D_{27}^{1, k}+D_{311}^{1, k}-D_{311}^{3, k}-D_{312}^{5, k}\right)\right] \\
& +(t \rightarrow b)
\end{aligned}
$$




$$
\begin{aligned}
& f_{3}^{b, \hat{t}}=\frac{i g_{s}^{2}}{16 \pi^{2}} \sum_{k=1,2} F_{2, k}\left\{2 \left(D_{26}^{1, k}+D_{310}^{1, k}+D_{37}^{1, k}-D_{25}^{1, k}-D_{35}^{1, k}-D_{39}^{1, k}+D_{37}^{5, k}+D_{38}^{5, k}-D_{310}^{5, k}\right.\right. \\
& \left.-\quad D_{39}^{5, k}\right) p_{1} \cdot p_{2}+2\left(D_{22}^{1, k}+D_{25}^{1, k}+D_{35}^{1, k}+D_{36}^{1, k}+D_{39}^{1, k}-D_{24}^{1, k}-D_{26}^{1, k}-D_{34}^{1, k}-D_{37}^{1, k}\right. \\
& \left.-D_{38}^{1, k}+2 D_{310}^{5, k}+D_{26}^{5, k}+D_{39}^{5, k}-D_{25}^{5, k}-D_{35}^{5, k}-D_{37}^{5, k}-D_{38}^{5, k}\right) p_{1} \cdot p_{3}+2\left(D_{25}^{1, k}+D_{310}^{1, k}\right. \\
& +D_{39}^{1, k}-D_{26}^{1, k}-D_{37}^{1, k}-D_{38}^{1, k}+3 D_{310}^{5, k}-2 D_{36}^{5, k}-2 D_{38}^{5, k}+D_{32}^{5, k}+D_{34}^{5, k}+D_{39}^{5, k}-D_{35}^{5, k} \\
& \left.-\quad D_{37}^{5, k}\right) p_{2} \cdot p_{3}+\left(D_{12}^{1, k}-D_{11}^{1, k}+D_{12}^{5, k}-D_{11}^{5, k}\right) m_{t}^{2}+\left(D_{11}^{1, k}+D_{31}^{1, k}+2 D_{21}^{1, k}+2 D_{26}^{1, k}\right. \\
& +2 D_{310}^{1, k}+2 D_{37}^{1, k}-D_{12}^{1, k}-D_{34}^{1, k}-2 D_{24}^{1, k}-2 D_{25}^{1, k}-2 D_{35}^{1, k}-2 D_{39}^{1, k}+D_{36}^{5, k}-D_{32}^{5, k} \\
& \left.+2 D_{37}^{5, k}+2 D_{38}^{5, k}-2 D_{310}^{5, k}-2 D_{39}^{5, k}\right) m_{\tilde{\chi}_{1}^{+}}^{2} \\
& \left.+4\left(D_{312}^{1, k}-D_{311}^{1, k}+D_{312}^{5, k}-D_{311}^{5, k}\right)+2\left(D_{312}^{3, k}-D_{311}^{3, k}\right)\right\}+(t \rightarrow b) \\
& f_{4}^{b, \hat{t}}=\frac{i g_{s}^{2}}{16 \pi^{2}} \sum_{k=1,2} F_{2, k}\left\{2 \left(D_{26}^{1, k}+D_{310}^{1, k}+D_{33}^{1, k}-D_{23}^{1, k}-D_{37}^{1, k}-D_{39}^{1, k}+D_{23}^{5, k}+D_{37}^{5, k}-D_{26}^{5, k}\right.\right. \\
& \left.-D_{310}^{5, k}\right) p_{1} \cdot p_{2}+2\left[2\left(D_{23}^{1, k}+D_{39}^{1, k}-D_{26}^{1, k}-D_{310}^{1, k}\right)+D_{22}^{1, k}+D_{36}^{1, k}+D_{37}^{1, k}-D_{25}^{1, k}-D_{33}^{1, k}\right. \\
& \left.-D_{38}^{1, k}+D_{26}^{5, k}+D_{310}^{5, k}-D_{13}^{5, k}-D_{23}^{5, k}-D_{35}^{5, k}-D_{37}^{5, k}-2 D_{25}^{5, k}\right] p_{1} \cdot p_{3}+2\left(2 D_{39}^{1, k}+D_{23}^{1, k}\right. \\
& -D_{26}^{1, k}-D_{33}^{1, k}-D_{38}^{1, k}+2 D_{26}^{5, k}+2 D_{310}^{5, k}+D_{24}^{5, k}+D_{34}^{5, k}-D_{22}^{5, k}-D_{23}^{5, k}-D_{25}^{5, k}-D_{35}^{5, k} \\
& \left.-\quad D_{36}^{5, k}-D_{37}^{5, k}\right) p_{2} \cdot p_{3}+\left(D_{12}^{1, k}-D_{13}^{1, k}-D_{0}^{5, k}-D_{11}^{5, k}\right) m_{t}^{2}+\left[2 \left(D_{25}^{1, k}+D_{26}^{1, k}+D_{310}^{1, k}\right.\right. \\
& \left.+D_{33}^{1, k}-D_{23}^{1, k}-D_{24}^{1, k}-D_{37}^{1, k}-D_{39}^{1, k}+D_{23}^{5, k}+D_{37}^{5, k}-D_{26}^{5, k}-D_{310}^{5, k}\right)+D_{13}^{1, k}+D_{35}^{1, k} \\
& \left.-D_{12}^{1, k}-D_{34}^{1, k}+D_{22}^{5, k}+D_{36}^{5, k}\right] m_{\tilde{\chi}_{1}^{+}}^{2}+4 D_{312}^{1, k}+2 D_{27}^{1, k}-6 D_{313}^{1, k} \\
& \left.+2 D_{27}^{3, k}+2 D_{312}^{3, k}-2 D_{27}^{5, k}-4 D_{311}^{5, k}\right\}+(t \rightarrow b) \\
& f_{5}^{b, \hat{t}}=\frac{i g_{s}^{2}}{16 \pi^{2}} \sum_{k=1,2}\left\{2 m_{t} m_{\tilde{\chi}_{1}^{+}} F_{1, k}\left(D_{13}^{1, k}-D_{0}^{1, k}-D_{11}^{1, k}\right)+F_{2, k}\left[2\left(D_{26}^{1, k}-D_{25}^{1, k}\right) p_{2} \cdot p_{3}-D_{0}^{1, k} m_{t}^{2}\right.\right.
\end{aligned}
$$




$$
\begin{aligned}
& +\left(2 D_{13}^{1, k}+2 D_{25}^{1, k}-D_{0}^{1, k}-D_{21}^{1, k}-2 D_{11}^{1, k}\right) m_{\tilde{\chi}_{1}^{+}}^{2}+2\left(D_{311}^{1, k}-D_{313}^{1, k}+D_{313}^{3, k}-D_{27}^{3, k}-D_{311}^{3, k}\right. \\
& \left.\left.\left.-\quad D_{313}^{5, k}\right)\right]\right\}+(t \rightarrow b) \\
& f_{6}^{b, \hat{t}}=\frac{i g_{s}^{2}}{16 \pi^{2}} \sum_{k=1,2}\left\{2 D_{13}^{1, k} m_{t} m_{\tilde{\chi}_{1}^{+}} F_{1, k}+F_{2, k}\left[2\left(D_{37}^{1, k}-D_{33}^{1, k}\right) p_{1} \cdot p_{2}+2\left(D_{25}^{1, k}+D_{310}^{1, k}+D_{33}^{1, k}\right.\right.\right. \\
& \left.-\quad D_{23}^{1, k}-D_{37}^{1, k}-D_{39}^{1, k}\right) p_{1} \cdot p_{3}+2\left(D_{33}^{1, k}-D_{39}^{1, k}\right) p_{2} \cdot p_{3}+D_{13}^{1, k} m_{t}^{2}+\left(D_{13}^{1, k}+2 D_{37}^{1, k}-D_{35}^{1, k}\right. \\
& \left.\left.\left.-\quad 2 D_{33}^{1, k}\right) m_{\tilde{\chi}_{1}^{+}}^{2}+4 D_{313}^{1, k}+2 D_{313}^{3, k}+2 D_{312}^{5, k}-2 D_{313}^{5, k}\right]\right\}+(t \rightarrow b) \\
& f_{7}^{b, \hat{t}}=\frac{i g_{s}^{2}}{8 \pi^{2}} \sum_{k=1,2}\left[m _ { t } F _ { 1 , k } \left(D_{12}^{1, k}+D_{24}^{1, k}+D_{25}^{1, k}+D_{12}^{3, k}+D_{24}^{3, k}+D_{25}^{3, k}+D_{25}^{5, k}-D_{11}^{1, k}-D_{21}^{1, k}\right.\right. \\
& \left.-D_{26}^{1, k}-D_{11}^{3, k}-D_{21}^{3, k}-D_{26}^{3, k}-D_{26}^{5, k}\right)+m_{\tilde{\chi}_{1}^{+}} F_{2, k}\left(2 D_{24}^{1, k}+D_{12}^{1, k}+D_{25}^{1, k}+D_{34}^{1, k}+D_{35}^{1, k}\right. \\
& +D_{21}^{3, k}+D_{310}^{3, k}+D_{31}^{3, k}+D_{38}^{5, k}-2 D_{21}^{1, k}-D_{11}^{1, k}-D_{26}^{1, k}-D_{310}^{1, k}-D_{31}^{1, k}-D_{24}^{3, k}-D_{34}^{3, k} \\
& \left.\left.-\quad D_{35}^{3, k}-D_{310}^{5, k}\right)\right]+(t \rightarrow b) \\
& f_{8}^{b, \hat{t}}=\frac{i g_{s}^{2}}{8 \pi^{2}} \sum_{k=1,2}\left[m _ { t } F _ { 1 , k } \left(D_{12}^{1, k}+D_{24}^{1, k}+D_{0}^{3, k}+D_{11}^{3, k}+D_{12}^{3, k}+D_{24}^{3, k}+D_{13}^{5, k}+D_{25}^{5, k}-D_{26}^{1, k}\right.\right. \\
& \left.-D_{13}^{3, k}-D_{26}^{3, k}\right)+m_{\tilde{\chi}_{1}^{+}} F_{2, k}\left(2 D_{24}^{1, k}+D_{12}^{1, k}+D_{34}^{1, k}+D_{25}^{3, k}+D_{310}^{3, k}-D_{26}^{1, k}-D_{310}^{1, k}-D_{11}^{3, k}\right. \\
& \left.\left.-D_{21}^{3, k}-D_{24}^{3, k}-D_{34}^{3, k}-D_{26}^{5, k}-D_{310}^{5, k}\right)\right]+(t \rightarrow b) \\
& f_{9}^{b, \hat{t}}=\frac{i g_{s}^{2}}{8 \pi^{2}} \sum_{k=1,2}\left[m_{t} F_{1, k}\left(D_{25}^{1, k}+D_{25}^{3, k}+D_{22}^{5, k}+D_{25}^{5, k}-D_{26}^{1, k}-D_{26}^{3, k}-D_{24}^{5, k}-D_{26}^{5, k}\right)\right. \\
& \left.+m_{\tilde{\chi}_{1}^{+}} F_{2, k}\left(D_{25}^{1, k}+D_{35}^{1, k}+D_{310}^{3, k}+D_{36}^{5, k}+D_{38}^{5, k}-D_{26}^{1, k}-D_{310}^{1, k}-D_{35}^{3, k}-D_{310}^{5, k}-D_{32}^{5, k}\right)\right] \\
& +(t \rightarrow b) \\
& f_{10}^{b, \hat{t}}=\frac{i g_{s}^{2}}{8 \pi^{2}} \sum_{k=1,2}\left[m_{t} F_{1, k}\left(D_{13}^{5, k}+D_{25}^{5, k}-D_{26}^{1, k}-D_{13}^{3, k}-D_{26}^{3, k}-D_{12}^{5, k}-D_{24}^{5, k}\right)\right. \\
& \left.+m_{\tilde{\chi}_{1}^{+}} F_{2, k}\left(D_{25}^{3, k}+D_{310}^{3, k}+D_{22}^{5, k}+D_{36}^{5, k}-D_{26}^{1, k}-D_{310}^{1, k}-D_{26}^{5, k}-D_{310}^{5, k}\right)\right]+(t \rightarrow b)
\end{aligned}
$$




$$
\begin{aligned}
& f_{11}^{b, \hat{t}}=\frac{i g_{s}^{2}}{32 \pi^{2}} \sum_{k=1,2}\left(-2 D_{0}^{1, k} m_{t} m_{\tilde{\chi}_{1}^{+}} F_{1, k}+F_{2, k}\left\{2 \left(D_{26}^{1, k}+D_{310}^{1, k}+D_{33}^{1, k}-D_{25}^{1, k}-D_{37}^{1, k}\right.\right.\right. \\
& \left.-\quad D_{39}^{1, k}\right) p_{1} \cdot p_{2}+2\left(D_{22}^{1, k}+D_{23}^{1, k}+D_{36}^{1, k}+D_{37}^{1, k}+2 D_{39}^{1, k}-D_{33}^{1, k}-D_{38}^{1, k}-2 D_{26}^{1, k}\right. \\
& \left.-2 D_{310}^{1, k}\right) p_{1} \cdot p_{3}+2\left(2 D_{39}^{1, k}-D_{33}^{1, k}-D_{38}^{1, k}\right) p_{2} \cdot p_{3}+\left(D_{12}^{1, k}-D_{0}^{1, k}-D_{13}^{1, k}\right) m_{t}^{2} \\
& +\left[2\left(D_{26}^{1, k}+D_{310}^{1, k}+D_{33}^{1, k}-D_{24}^{1, k}-D_{37}^{1, k}-D_{39}^{1, k}\right)+D_{13}^{1, k}+D_{21}^{1, k}+D_{35}^{1, k}-D_{0}^{1, k}\right. \\
& \left.-\quad D_{12}^{1, k}-D_{34}^{1, k}\right] m_{\tilde{\chi}_{1}^{+}}^{2}+4\left(D_{312}^{1, k}-D_{313}^{1, k}\right)+2\left(D_{27}^{5, k}+D_{311}^{5, k}\right. \\
& \left.\left.\left.+D_{313}^{5, k}+D_{312}^{3, k}-D_{313}^{3, k}-D_{312}^{5, k}\right)\right\}\right)+(t \rightarrow b) \\
& f_{12}^{b, \hat{t}}=\frac{i g_{s}^{2}}{16 \pi^{2}} \sum_{k=1,2} F_{2, k}\left(D_{313}^{1, k}+D_{311}^{5, k}+D_{313}^{5, k}+D_{312}^{3, k}-D_{27}^{1, k}-D_{312}^{1, k}-D_{313}^{3, k}-D_{312}^{5, k}\right)+(t \rightarrow b) \\
& f_{13}^{b, \hat{t}}=\frac{i g_{s}^{2}}{16 \pi^{2}} \sum_{k=1,2}\left[m_{t} F_{1, k}\left(D_{12}^{1, k}+D_{12}^{5, k}-D_{11}^{1, k}-D_{11}^{5, k}\right)\right. \\
& \left.+m_{\tilde{\chi}_{1}^{+}} F_{2, k}\left(D_{12}^{1, k}+D_{24}^{1, k}+D_{24}^{5, k}-D_{11}^{1, k}-D_{21}^{1, k}-D_{22}^{5, k}\right)\right]+(t \rightarrow b) \\
& f_{14}^{b, \hat{t}}=\frac{i g_{s}^{2}}{16 \pi^{2}} \sum_{k=1,2}\left[m_{t} F_{1, k}\left(D_{12}^{1, k}-D_{0}^{5, k}-D_{11}^{5, k}\right)+m_{\tilde{\chi}_{1}^{+}} F_{2, k}\left(D_{12}^{1, k}+D_{24}^{1, k}+D_{12}^{5, k}+D_{24}^{5, k}\right)\right] \\
& +(t \rightarrow b) \\
& f_{15}^{b, \hat{t}}=\frac{i g_{s}^{2}}{16 \pi^{2}} \sum_{k=1,2}\left[m_{t} F_{1, k}\left(D_{11}^{1, k}-D_{13}^{1, k}\right)+m_{\tilde{\chi}_{1}^{+}} F_{2, k}\left(D_{11}^{1, k}+D_{21}^{1, k}-D_{13}^{1, k}-D_{25}^{1, k}\right)\right]+(t \rightarrow b) \\
& f_{16}^{b, \hat{t}}=-\frac{i g_{s}^{2}}{16 \pi^{2}} \sum_{k=1,2}\left[D_{13}^{1, k} m_{t} F_{1, k}+m_{\tilde{\chi}_{1}^{+}} F_{2, k}\left(D_{13}^{1, k}+D_{25}^{1, k}\right)\right]+(t \rightarrow b) \\
& f_{17}^{b, \hat{t}}=\frac{i g_{s}^{2}}{8 \pi^{2}} \sum_{k=1,2} F_{2, k}\left(D_{24}^{1, k}+D_{26}^{1, k}+D_{34}^{1, k}+D_{37}^{1, k}+D_{38}^{1, k}+D_{22}^{3, k}+D_{25}^{3, k}+D_{35}^{3, k}+D_{36}^{3, k}\right. \\
& +D_{39}^{3, k}+D_{25}^{5, k}+D_{35}^{5, k}+D_{37}^{5, k}+D_{38}^{5, k}-D_{22}^{1, k}-D_{25}^{1, k}-D_{35}^{1, k}-D_{36}^{1, k}-D_{39}^{1, k}-D_{24}^{3, k} \\
& \left.-D_{26}^{3, k}-D_{34}^{3, k}-D_{37}^{3, k}-D_{38}^{3, k}-D_{26}^{5, k}-D_{39}^{5, k}-2 D_{310}^{5, k}\right)+(t \rightarrow b)
\end{aligned}
$$




$$
\begin{aligned}
& f_{18}^{b, \hat{t}}=\frac{i g_{s}^{2}}{8 \pi^{2}} \sum_{k=1,2} F_{2, k}\left(D_{25}^{1, k}+D_{26}^{1, k}+D_{310}^{1, k}+D_{38}^{1, k}+D_{12}^{3, k}+D_{22}^{3, k}+D_{23}^{3, k}+D_{24}^{3, k}+D_{36}^{3, k}\right. \\
& +D_{39}^{3, k}+2 D_{25}^{5, k}+D_{13}^{5, k}+D_{23}^{5, k}+D_{35}^{5, k}+D_{37}^{5, k}-D_{22}^{1, k}-D_{23}^{1, k}-D_{36}^{1, k}-D_{39}^{1, k}-2 D_{26}^{3, k} \\
& \left.-D_{13}^{3, k}-D_{25}^{3, k}-D_{310}^{3, k}-D_{38}^{3, k}-D_{26}^{5, k}-D_{310}^{5, k}\right)+(t \rightarrow b) \\
& f_{19}^{b, \hat{t}}=\frac{i g_{s}^{2}}{8 \pi^{2}} \sum_{k=1,2} F_{2, k}\left(D_{26}^{1, k}+D_{37}^{1, k}+D_{38}^{1, k}+D_{310}^{3, k}+D_{39}^{3, k}+2 D_{36}^{5, k}+2 D_{38}^{5, k}+D_{35}^{5, k}+D_{37}^{5, k}\right. \\
& \left.-D_{25}^{1, k}-D_{310}^{1, k}-D_{39}^{1, k}-D_{37}^{3, k}-D_{38}^{3, k}-3 D_{310}^{5, k}-D_{32}^{5, k}-D_{34}^{5, k}-D_{39}^{5, k}\right)+(t \rightarrow b) \\
& f_{20}^{b, \hat{t}}=\frac{i g_{s}^{2}}{8 \pi^{2}} \sum_{k=1,2} F_{2, k}\left(D_{26}^{1, k}+D_{38}^{1, k}+D_{23}^{3, k}+D_{39}^{3, k}+D_{22}^{5, k}+D_{23}^{5, k}+D_{25}^{5, k}+D_{35}^{5, k}+D_{36}^{5, k}\right. \\
& \left.+D_{37}^{5, k}-D_{24}^{5, k}-D_{23}^{1, k}-D_{39}^{1, k}-D_{26}^{3, k}-D_{38}^{3, k}-D_{34}^{5, k}-2 D_{26}^{5, k}-2 D_{310}^{5, k}\right)+(t \rightarrow b) \\
& f_{34}^{b, \hat{t}}=\frac{i g_{s}^{2}}{32 \pi^{2}} \sum_{k=1,2}\left(m _ { t } F _ { 3 , k } \left\{2\left(D_{13}^{1, k}+D_{25}^{1, k}-D_{23}^{1, k}\right) p_{1} \cdot p_{2}+2\left(D_{11}^{1, k}+D_{12}^{1, k}+D_{23}^{1, k}+D_{24}^{1, k}\right.\right.\right. \\
& \left.-D_{25}^{1, k}-D_{26}^{1, k}-2 D_{13}^{1, k}\right) p_{1} \cdot p_{3}+2\left(D_{23}^{1, k}-D_{13}^{1, k}-D_{26}^{1, k}\right) p_{2} \cdot p_{3}+\left[2 \left(D_{13}^{1, k}+D_{25}^{1, k}\right.\right. \\
& \left.\left.\left.-\quad D_{11}^{1, k}-D_{23}^{1, k}\right)-D_{0}^{1, k}-D_{21}^{1, k}\right] m_{\tilde{\chi}_{1}^{+}}^{2}+4 D_{27}^{1, k}-2\left(D_{27}^{5, k}+D_{27}^{1, k}+D_{27}^{3, k}\right)+D_{0}^{1, k} m_{t}^{2}\right\} \\
& +m_{\tilde{\chi}_{1}^{+}} F_{4, k}\left[2\left(D_{35}^{1, k}+2 D_{33}^{1, k}-3 D_{37}^{1, k}-D_{13}^{1, k}-D_{23}^{1, k}\right) p_{1} \cdot p_{2}+2\left(3 D_{23}^{1, k}+3 D_{37}^{1, k}+D_{11}^{1, k}\right.\right. \\
& \left.+D_{21}^{1, k}+D_{34}^{1, k}+2 D_{39}^{1, k}-3 D_{25}^{1, k}-3 D_{310}^{1, k}-D_{12}^{1, k}-D_{26}^{1, k}-D_{35}^{1, k}-2 D_{33}^{1, k}\right) p_{1} \cdot p_{3} \\
& +2\left(D_{13}^{1, k}+D_{23}^{1, k}+D_{26}^{1, k}+D_{37}^{1, k}+2 D_{39}^{1, k}-D_{25}^{1, k}-D_{310}^{1, k}-2 D_{33}^{1, k}\right) p_{2} \cdot p_{3}+\left(D_{11}^{1, k}-2 D_{13}^{1, k}\right. \\
& \left.-D_{0}^{1, k}\right) m_{t}^{2}+4\left(D_{311}^{1, k}+D_{313}^{5, k}-2 D_{313}^{1, k}-D_{313}^{3, k}\right)+2\left(D_{311}^{3, k}-D_{312}^{5, k}\right) \\
& \left.\left.+\left(D_{0}^{1, k}+D_{11}^{1, k}+4 D_{25}^{1, k}+4 D_{33}^{1, k}+4 D_{35}^{1, k}-6 D_{37}^{1, k}-2 D_{23}^{1, k}-D_{21}^{1, k}-D_{31}^{1, k}\right) m_{\tilde{\chi}_{1}^{+}}^{2}\right]\right) \\
& +(t \rightarrow b) \\
& f_{35}^{b, \hat{t}}=\frac{i g_{s}^{2}}{16 \pi^{2}} \sum_{k=1,2}\left\{-m_{t} F_{3, k}\left(D_{27}^{1, k}+D_{27}^{3, k}+D_{27}^{5, k}\right)+m_{\tilde{\chi}_{1}^{+}} F_{4, k}\left[2\left(D_{313}^{1, k}+D_{313}^{5, k}-D_{313}^{3, k}\right)\right.\right.
\end{aligned}
$$




$$
\begin{aligned}
& \left.\left.+\quad D_{311}^{3, k}-D_{27}^{1, k}-D_{311}^{1, k}-D_{312}^{5, k}\right]\right\}+(t \rightarrow b) \\
& f_{36}^{b, \hat{t}}=\frac{i g_{s}^{2}}{16 \pi^{2}} \sum_{k=1,2} F_{4, k}\left\{2 \left(D_{26}^{1, k}+D_{310}^{1, k}+D_{37}^{1, k}+D_{37}^{5, k}+D_{38}^{5, k}-D_{25}^{1, k}-D_{35}^{1, k}-D_{39}^{1, k}-D_{310}^{5, k}\right.\right. \\
& \left.-D_{39}^{5, k}\right) p_{1} \cdot p_{2}+2\left(D_{22}^{1, k}+D_{25}^{1, k}+D_{35}^{1, k}+D_{36}^{1, k}+D_{39}^{1, k}+2 D_{310}^{5, k}+D_{26}^{5, k}+D_{39}^{5, k}-D_{24}^{1, k}\right. \\
& \left.-D_{26}^{1, k}-D_{34}^{1, k}-D_{37}^{1, k}-D_{38}^{1, k}-D_{25}^{5, k}-D_{35}^{5, k}-D_{37}^{5, k}-D_{38}^{5, k}\right) p_{1} \cdot p_{3}+2\left(D_{25}^{1, k}+D_{310}^{1, k}\right. \\
& +D_{39}^{1, k}+3 D_{310}^{5, k}+D_{32}^{5, k}+D_{34}^{5, k}+D_{39}^{5, k}-D_{26}^{1, k}-D_{37}^{1, k}-D_{38}^{1, k}-2 D_{36}^{5, k}-2 D_{38}^{5, k}-D_{35}^{5, k} \\
& \left.-D_{37}^{5, k}\right) p_{2} \cdot p_{3}+\left(D_{12}^{1, k}+D_{12}^{5, k}-D_{11}^{1, k}-D_{11}^{5, k}\right) m_{t}^{2}+\left[D_{11}^{1, k}+D_{31}^{1, k}-D_{12}^{1, k}-D_{34}^{1, k}+D_{36}^{5, k}\right. \\
& -D_{32}^{5, k}+2\left(D_{21}^{1, k}+D_{26}^{1, k}+D_{310}^{1, k}+D_{37}^{1, k}+D_{37}^{5, k}+D_{38}^{5, k}-D_{24}^{1, k}-D_{25}^{1, k}-D_{35}^{1, k}-D_{39}^{1, k}\right. \\
& \left.\left.-D_{310}^{5, k}-D_{39}^{5, k}\right)\right] m_{\tilde{\chi}_{1}^{+}}^{2}+4\left(D_{312}^{1, k}+D_{312}^{5, k}-D_{311}^{1, k}-D_{311}^{5, k}\right) \\
& \left.+2\left(D_{312}^{3, k}-D_{311}^{3, k}\right)\right\}+(t \rightarrow b) \\
& f_{37}^{b, \hat{t}}=\frac{i g_{s}^{2}}{16 \pi^{2}} \sum_{k=1,2} F_{4, k}\left\{2 \left(D_{26}^{1, k}+D_{310}^{1, k}+D_{33}^{1, k}+D_{23}^{5, k}+D_{37}^{5, k}-D_{23}^{1, k}-D_{37}^{1, k}-D_{39}^{1, k}-D_{26}^{5, k}\right.\right. \\
& \left.-D_{310}^{5, k}\right) p_{1} \cdot p_{2}+2\left[2\left(D_{23}^{1, k}+D_{39}^{1, k}-D_{26}^{1, k}-D_{310}^{1, k}\right)+D_{22}^{1, k}+D_{36}^{1, k}+D_{37}^{1, k}+D_{26}^{5, k}+D_{310}^{5, k}\right. \\
& \left.-D_{25}^{1, k}-D_{33}^{1, k}-D_{38}^{1, k}-D_{13}^{5, k}-D_{23}^{5, k}-D_{35}^{5, k}-D_{37}^{5, k}-2 D_{25}^{5, k}\right] p_{1} \cdot p_{3}+2\left(2 D_{39}^{1, k}+D_{23}^{1, k}\right. \\
& +2 D_{26}^{5, k}+2 D_{310}^{5, k}+D_{24}^{5, k}+D_{34}^{5, k}-D_{26}^{1, k}-D_{33}^{1, k}-D_{38}^{1, k}-D_{22}^{5, k}-D_{23}^{5, k}-D_{25}^{5, k}-D_{35}^{5, k} \\
& \left.-D_{36}^{5, k}-D_{37}^{5, k}\right) p_{2} \cdot p_{3}+\left(D_{12}^{1, k}-D_{13}^{1, k}-D_{0}^{5, k}-D_{11}^{5, k}\right) m_{t}^{2}+\left[2 \left(D_{25}^{1, k}+D_{26}^{1, k}+D_{310}^{1, k}+D_{33}^{1, k}\right.\right. \\
& \left.+D_{23}^{5, k}+D_{37}^{5, k}-D_{23}^{1, k}-D_{24}^{1, k}-D_{37}^{1, k}-D_{39}^{1, k}-D_{26}^{5, k}-D_{310}^{5, k}\right)+D_{13}^{1, k}+D_{35}^{1, k}+D_{22}^{5, k} \\
& \left.+D_{36}^{5, k}-D_{12}^{1, k}-D_{34}^{1, k}\right] m_{\tilde{\chi}_{1}^{+}}^{2}-6 D_{313}^{1, k}+4\left(D_{312}^{1, k}-D_{311}^{5, k}\right) \\
& \left.+2\left(D_{27}^{1, k}+D_{27}^{3, k}+D_{312}^{3, k}-D_{27}^{5, k}\right)\right\}+(t \rightarrow b) \\
& f_{38}^{b, \hat{t}}=\frac{i g_{s}^{2}}{16 \pi^{2}} \sum_{k=1,2} F_{4, k}\left[2\left(D_{26}^{1, k}-D_{25}^{1, k}\right) p_{2} \cdot p_{3}-D_{0}^{1, k} m_{t}^{2}+\left(D_{0}^{1, k}+D_{21}^{1, k}+2 D_{11}^{1, k}\right) m_{\tilde{\chi}_{1}^{+}}^{2}\right.
\end{aligned}
$$




$$
\begin{aligned}
& \left.+2\left(D_{311}^{1, k}+D_{313}^{3, k}-D_{313}^{1, k}-D_{27}^{3, k}-D_{311}^{3, k}-D_{313}^{5, k}\right)\right]+(t \rightarrow b) \\
& f_{39}^{b, \hat{t}}=\frac{i g_{s}^{2}}{16 \pi^{2}} \sum_{k=1,2} F_{4, k}\left[2\left(D_{37}^{1, k}-D_{33}^{1, k}\right) p_{1} \cdot p_{2}+2\left(D_{25}^{1, k}+D_{310}^{1, k}+D_{33}^{1, k}-D_{23}^{1, k}-D_{37}^{1, k}\right.\right. \\
& \left.-\quad D_{39}^{1, k}\right) p_{1} \cdot p_{3}+2\left(D_{33}^{1, k}-D_{39}^{1, k}\right) p_{2} \cdot p_{3}+D_{13}^{1, k} m_{t}^{2}+\left(2 D_{37}^{1, k}-2 D_{25}^{1, k}-2 D_{33}^{1, k}-D_{13}^{1, k}\right. \\
& \left.\left.-\quad D_{35}^{1, k}\right) m_{\tilde{\chi}_{1}^{+}}^{2}+4 D_{313}^{1, k}+2\left(D_{313}^{3, k}+D_{312}^{5, k}-D_{313}^{5, k}\right)\right]+(t \rightarrow b) \\
& f_{40}^{b, \hat{t}}=\frac{i g_{s}^{2}}{8 \pi^{2}} \sum_{k=1,2}\left\{m _ { t } F _ { 3 , k } \left(D_{11}^{1, k}+D_{21}^{1, k}+D_{26}^{1, k}+D_{11}^{3, k}+D_{21}^{3, k}+D_{26}^{3, k}+D_{26}^{5, k}-D_{12}^{1, k}-D_{24}^{1, k}\right.\right. \\
& \left.-D_{25}^{1, k}-D_{12}^{3, k}-D_{24}^{3, k}-D_{25}^{3, k}-D_{25}^{5, k}\right)+m_{\tilde{\chi}_{1}^{+}} F_{4, k}\left[3 \left(D_{26}^{1, k}+D_{310}^{1, k}+D_{35}^{3, k}-D_{25}^{1, k}-D_{35}^{1, k}\right.\right. \\
& \left.-D_{310}^{3, k}\right)+2\left(D_{21}^{1, k}+D_{37}^{1, k}+D_{25}^{3, k}+D_{39}^{3, k}+D_{37}^{5, k}-D_{24}^{1, k}-D_{39}^{1, k}-D_{26}^{3, k}-D_{37}^{3, k}-D_{39}^{5, k}\right) \\
& \left.\left.+D_{11}^{1, k}+D_{31}^{1, k}+D_{24}^{3, k}+D_{34}^{3, k}+D_{38}^{5, k}-D_{12}^{1, k}-D_{34}^{1, k}-D_{21}^{3, k}-D_{31}^{3, k}-D_{310}^{5, k}\right]\right\}+(t \rightarrow b) \\
& f_{41}^{b, \hat{t}}=\frac{i g_{s}^{2}}{8 \pi^{2}} \sum_{k=1,2}\left\{m _ { t } F _ { 3 , k } \left(D_{26}^{1, k}+D_{13}^{3, k}+D_{26}^{3, k}-D_{12}^{1, k}-D_{24}^{1, k}-D_{0}^{3, k}-D_{11}^{3, k}-D_{12}^{3, k}-D_{24}^{3, k}\right.\right. \\
& \left.-D_{13}^{5, k}-D_{25}^{5, k}\right)+m_{\tilde{\chi}_{1}^{+}} F_{4, k}\left[3\left(D_{26}^{1, k}+D_{310}^{1, k}-D_{25}^{3, k}-D_{310}^{3, k}\right)+2\left(D_{13}^{1, k}+D_{25}^{1, k}+D_{23}^{3, k}\right.\right. \\
& \left.+D_{39}^{3, k}+D_{23}^{5, k}+D_{37}^{5, k}-D_{23}^{1, k}-D_{24}^{1, k}-D_{39}^{1, k}-D_{13}^{3, k}-D_{26}^{3, k}\right)+D_{11}^{3, k}+D_{21}^{3, k}+D_{24}^{3, k} \\
& \left.\left.+\quad D_{34}^{3, k}-D_{12}^{1, k}-D_{34}^{1, k}-D_{26}^{5, k}-D_{310}^{5, k}\right]\right\}+(t \rightarrow b) \\
& f_{42}^{b, \hat{t}}=\frac{i g_{s}^{2}}{8 \pi^{2}} \sum_{k=1,2}\left\{m_{t} F_{3, k}\left(D_{26}^{1, k}+D_{26}^{3, k}+D_{24}^{5, k}+D_{26}^{5, k}-D_{25}^{1, k}-D_{25}^{3, k}-D_{22}^{5, k}-D_{25}^{5, k}\right)\right. \\
& +m_{\tilde{\chi}_{1}^{+}} F_{4, k}\left[3\left(D_{38}^{5, k}-D_{310}^{5, k}\right)+2\left(D_{37}^{1, k}+D_{39}^{3, k}+D_{37}^{5, k}-D_{39}^{1, k}-D_{37}^{3, k}-D_{39}^{5, k}\right)+D_{26}^{1, k}\right. \\
& \left.\left.+D_{310}^{1, k}+D_{35}^{3, k}+D_{36}^{5, k}-D_{25}^{1, k}-D_{35}^{1, k}-D_{310}^{3, k}-D_{32}^{5, k}\right]\right\}+(t \rightarrow b) \\
& f_{43}^{b, \hat{t}}=\frac{i g_{s}^{2}}{8 \pi^{2}} \sum_{k=1,2}\left\{m_{t} F_{3, k}\left(D_{26}^{1, k}+D_{13}^{3, k}+D_{26}^{3, k}+D_{12}^{5, k}+D_{24}^{5, k}-D_{13}^{5, k}-D_{25}^{5, k}\right)+m_{\tilde{\chi}_{1}^{+}} F_{4, k}\left[D_{26}^{1, k}\right.\right.
\end{aligned}
$$




$$
\begin{aligned}
& +D_{310}^{1, k}+D_{22}^{5, k}+D_{36}^{5, k}-D_{25}^{3, k}-D_{310}^{3, k}+2\left(D_{23}^{3, k}+D_{39}^{3, k}+D_{23}^{5, k}+D_{37}^{5, k}-D_{23}^{1, k}-D_{39}^{1, k}\right) \\
& \left.\left.-\quad 3\left(D_{26}^{5, k}+D_{310}^{5, k}\right)\right]\right\}+(t \rightarrow b) \\
& f_{44}^{b, \hat{t}}=\frac{i g_{s}^{2}}{32 \pi^{2}} \sum_{k=1,2} F_{4, k}\left\{2\left(D_{26}^{1, k}+D_{310}^{1, k}+D_{33}^{1, k}-D_{25}^{1, k}-D_{37}^{1, k}-D_{39}^{1, k}\right) p_{1} \cdot p_{2}+2\left(D_{22}^{1, k}+D_{23}^{1, k}\right.\right. \\
& \left.+D_{36}^{1, k}+D_{37}^{1, k}-D_{33}^{1, k}-D_{38}^{1, k}+2 D_{39}^{1, k}-2 D_{26}^{1, k}-2 D_{310}^{1, k}\right) p_{1} \cdot p_{3}+2\left(2 D_{39}^{1, k}-D_{33}^{1, k}\right. \\
& \left.-D_{38}^{1, k}\right) p_{2} \cdot p_{3}+\left(D_{12}^{1, k}-D_{0}^{1, k}-D_{13}^{1, k}\right) m_{t}^{2}+\left[2 \left(D_{11}^{1, k}+D_{26}^{1, k}+D_{310}^{1, k}+D_{33}^{1, k}-D_{24}^{1, k}-D_{37}^{1, k}\right.\right. \\
& \left.\left.-D_{39}^{1, k}\right)+D_{0}^{1, k}+D_{13}^{1, k}+D_{21}^{1, k}+D_{35}^{1, k}-D_{12}^{1, k}-D_{34}^{1, k}\right] m_{\tilde{\chi}_{1}^{+}}^{2}+4\left(D_{312}^{1, k}\right. \\
& \left.\left.-\quad D_{313}^{1, k}\right)+2\left(D_{27}^{5, k}+D_{311}^{5, k}+D_{313}^{5, k}+D_{312}^{3, k}-D_{313}^{3, k}-D_{312}^{5, k}\right)\right\}+(t \rightarrow b) \\
& f_{45}^{b, \hat{t}}=\frac{i g_{s}^{2}}{16 \pi^{2}} \sum_{k=1,2} F_{4, k}\left(D_{313}^{1, k}+D_{312}^{3, k}+D_{311}^{5, k}+D_{313}^{5, k}-D_{27}^{1, k}-D_{312}^{1, k}-D_{313}^{3, k}-D_{312}^{5, k}\right)+(t \rightarrow b) \\
& f_{46}^{b, \hat{t}}=\frac{i g_{s}^{2}}{16 \pi^{2}} \sum_{k=1,2}\left\{m_{t} F_{3, k}\left(D_{11}^{1, k}+D_{11}^{5, k}-D_{12}^{1, k}-D_{12}^{5, k}\right)+m_{\tilde{\chi}_{1}^{+}} F_{4, k}\left[2 \left(D_{26}^{1, k}+D_{26}^{5, k}-D_{25}^{1, k}\right.\right.\right. \\
& \left.\left.\left.-D_{25}^{5, k}\right)+D_{11}^{1, k}+D_{21}^{1, k}+D_{24}^{5, k}-D_{12}^{1, k}-D_{24}^{1, k}-D_{22}^{5, k}\right]\right\}+(t \rightarrow b) \\
& f_{47}^{b, \hat{t}}=\frac{i g_{s}^{2}}{16 \pi^{2}} \sum_{k=1,2}\left\{m_{t} F_{3, k}\left(D_{0}^{5, k}+D_{11}^{5, k}-D_{12}^{1, k}\right)+m_{\tilde{\chi}_{1}^{+}} F_{4, k}\left[2\left(D_{13}^{1, k}+D_{26}^{1, k}-D_{13}^{5, k}-D_{25}^{5, k}\right)\right.\right. \\
& \left.\left.+D_{12}^{5, k}+D_{24}^{5, k}-D_{12}^{1, k}-D_{24}^{1, k}\right]\right\}+(t \rightarrow b) \\
& f_{48}^{b, \hat{t}}=\frac{i g_{s}^{2}}{16 \pi^{2}} \sum_{k=1,2}\left\{m_{t} F_{3, k}\left(D_{13}^{1, k}-D_{11}^{1, k}\right)+m_{\tilde{\chi}_{1}^{+}} F_{4, k}\left[2\left(D_{12}^{1, k}+D_{24}^{1, k}-D_{26}^{1, k}\right)+D_{25}^{1, k}-D_{11}^{1, k}\right.\right. \\
& \left.\left.-\quad D_{13}^{1, k}-D_{21}^{1, k}\right]\right\}+(t \rightarrow b) \\
& f_{49}^{b, \hat{t}}=\frac{i g_{s}^{2}}{16 \pi^{2}} \sum_{k=1,2}\left[D_{13}^{1, k} m_{t} F_{3, k}+m_{\tilde{\chi}_{1}^{+}} F_{4, k}\left(D_{25}^{1, k}-D_{13}^{1, k}-2 D_{26}^{1, k}\right)\right]+(t \rightarrow b)
\end{aligned}
$$




$$
\begin{aligned}
& f_{50}^{b, \hat{t}}=\frac{i g_{s}^{2}}{8 \pi^{2}} \sum_{k=1,2} F_{4, k}\left(D_{24}^{1, k}+D_{26}^{1, k}+D_{34}^{1, k}+D_{37}^{1, k}+D_{38}^{1, k}+D_{22}^{3, k}+D_{25}^{3, k}+D_{35}^{3, k}+D_{36}^{3, k}+D_{39}^{3, k}\right. \\
& +D_{25}^{5, k}+D_{35}^{5, k}+D_{37}^{5, k}+D_{38}^{5, k}-D_{22}^{1, k}-D_{25}^{1, k}-D_{35}^{1, k}-D_{36}^{1, k}-D_{39}^{1, k}-D_{24}^{3, k}-D_{26}^{3, k}-D_{34}^{3, k} \\
& \left.-D_{37}^{3, k}-D_{38}^{3, k}-D_{26}^{5, k}-D_{39}^{5, k}-2 D_{310}^{5, k}\right)+(t \rightarrow b) \\
& f_{51}^{b, \hat{t}}=\frac{i g_{s}^{2}}{8 \pi^{2}} \sum_{k=1,2} F_{4, k}\left(D_{25}^{1, k}+D_{26}^{1, k}+D_{310}^{1, k}+D_{38}^{1, k}+D_{12}^{3, k}+D_{22}^{3, k}+D_{23}^{3, k}+D_{24}^{3, k}+D_{36}^{3, k}+D_{39}^{3, k}\right. \\
& +D_{13}^{5, k}+D_{23}^{5, k}+D_{35}^{5, k}+D_{37}^{5, k}+2 D_{25}^{5, k}-D_{22}^{1, k}-D_{23}^{1, k}-D_{36}^{1, k}-D_{39}^{1, k}-2 D_{26}^{3, k}-D_{13}^{3, k}-D_{25}^{3, k} \\
& \left.-D_{310}^{3, k}-D_{38}^{3, k}-D_{26}^{5, k}-D_{310}^{5, k}\right)+(t \rightarrow b) \\
& f_{52}^{b, \hat{t}}=\frac{i g_{s}^{2}}{8 \pi^{2}} \sum_{k=1,2} F_{4, k}\left(D_{26}^{1, k}+D_{37}^{1, k}+D_{38}^{1, k}+D_{310}^{3, k}+D_{39}^{3, k}+D_{35}^{5, k}+D_{37}^{5, k}+2 D_{36}^{5, k}+2 D_{38}^{5, k}-D_{25}^{1, k}\right. \\
& \left.-D_{310}^{1, k}-D_{39}^{1, k}-D_{37}^{3, k}-D_{38}^{3, k}-D_{32}^{5, k}-D_{34}^{5, k}-D_{39}^{5, k}-3 D_{310}^{5, k}\right)+(t \rightarrow b) \\
& f_{53}^{b, \hat{t}}=\frac{i g_{s}^{2}}{8 \pi^{2}} \sum_{k=1,2} F_{4, k}\left(D_{26}^{1, k}+D_{38}^{1, k}+D_{23}^{3, k}+D_{39}^{3, k}+D_{22}^{5, k}+D_{23}^{5, k}+D_{25}^{5, k}+D_{35}^{5, k}+D_{36}^{5, k}+D_{37}^{5, k}\right. \\
& \left.-D_{23}^{1, k}-D_{39}^{1, k}-D_{26}^{3, k}-D_{38}^{3, k}-D_{24}^{5, k}-D_{34}^{5, k}-2 D_{26}^{5, k}-2 D_{310}^{5, k}\right)+(t \rightarrow b) \\
& f_{i}^{b, \hat{t}}=0 \quad(i=21 \sim 33,54 \sim 66)
\end{aligned}
$$

In this work we adopted the definitions of two-, three-, four-point one-loop PassarinoVeltman integral functions as shown in reference 25] and all the vector and tensor integrals can be deduced in the forms of scalar integrals [26].

\section{References}


[1] S.L. Glashow, Nucl. Phys. 22(1961)579; S. Weinberg, Phys. Rev. Lett. 1(1967)1264; A. Salam, Proc. 8th Nobel Symposium Stockholm 1968, ed. N. Svartholm(Almquist and Wiksells, Stockholm 1968) p.367; H.D. Politzer, Phys. Rep. 14(1974)129.

[2] P.W. Higgs, Phys. Lett 12(1964)132, Phys. Rev. Lett. 13 (1964)508; Phys.Rev. 145(1966)1156; F.Englert and R.Brout, Phys. Rev. Lett. 13(1964)321; G.S. Guralnik, C.R.Hagen and T.W.B. Kibble, Phys. Rev. Lett. 13(1964)585; T.W.B. Kibble, Phys. Rev. 155(1967)1554.

[3] H.E. Haber and G.L. Kane, Phys. Rep. 117, No.2-4, (1985)75.

[4] J.F. Gunion and H.E. Haber, Nucl. Phys. B272(1986)1.

[5] C.X. Chang, L. Han, W.G. Ma, et al, Nucl. Phys. B515 (1998)15; W. Beenakker, M. Krammer et al Nucl. Phys. B515(1998)3.

[6] M.A. Diaz, S.F. King and D.A. Ross, CERN-TH/97-313, SHEP-97/23, FTUV/97-47, IFIC/97-78, hep-ph/9711307.

[7] S.Y. Choi, A. Djouadi, H. Dreiner, J. Kalinowski, and P.M. Zerwas, 'Chargino Pair Production in $e^{+} e^{-}$Collisions', YUMS 98-10, SNUTP 98-52, DESY 98-77, PM 98-14, $\mathrm{IFT} / 9 / 98$.

[8] S. Kiyoura, M.M. Nojiri, D.M. Pierce and Y. Yamada, Phys. Rev. D58, 075002. 
[9] M.L. Zhou, W.G. Ma, L. Han, Y. Jiang and H. Zhou, 'The lightest chargino pair production via photon-photon collisions'(to be appeared in J. of Physics G), hepph/9903376.

[10] S.Y. Choi, A. Djouadi, H. Dreiner, J. Kalinowski, and P.M. Zerwas, 'Chargino Pair Production in $e^{+} e^{-}$Collisions', YUMS 98-10, SNUTP 98-52, DESY 98-77, PM 98-14, IFT/9/98. S. Kiyoura, M.M. Nojiri, D.M. Pierce and Y. Yamada, Phys. Rev. D58, 075002 .

[11] M. Acciarri et al, (L3 Collaboration), Eur. Phys. J. C4, (1998)207.

[12] K. Abbiendi et al, (OPAL Collaboration), Eur. Phys. J. C8, (1998)255.

[13] R. Barate et al, (ALEPH Collaboration), Eur. Phys. J. C2, (1998)417.

[14] P. Abreu et al, (DELPHI Collaboration), Eur. Phys. J. C1, (1998)1; Phys. Lett. B446, (1999)75.

[15] M.L. Zhou, W.G. Ma, L. Han, Y. Jiang and H. Zhou, J. of Phys. G25, 27(1999).

[16] S.Dimopoulos and Sutter, Nucl. Phys. B452(1995)496; H.Haber, Proceedings of the 5th International Conference on Supersymmetries in Physics(SUSY'97), May 1997, ed. M. Cvetic and P.Langacker, hep-ph/9709450.

[17] T. Ibrahim and P. Nath, Phys. Lett.B418(1998)98; Phys. Rev.D57(1998)478; Y. Kizukuri and N. Oshimo, Proceedings of the Workshop on $e^{+} e^{-}$Collisions at $500 \mathrm{GeV}$ : 
The Physics Potential, Munich-Annecy-Hamburg 1991/93, DES 92-123A+B, 93-123C, ed. P.Zerwas.

[18] S.Y. Choi, J.S. Shim, H.S. Song and W.Y. Song, YUMS 98-12, SNUTP 98-084, Aug. 1998.

[19] see for examples, J.R. Espinosa, and M Quiros, Phys. Lett B266 (1991)389; K. Gunion and A. Turski, Phys. Rev. D39 (1989)2701 and D40(1990)2333; M.Carena, M. Quiros and C.E.M. Wagner, Nucl. Phys.B461(1996)407.

[20] D.M. Copper, D.R.T. Jones and P.van Nieuwenhuizen, Nucl. Phys. B167(1980) 479; W. Siegel, Phys. Lett. B84(1979)193.

[21] G. Altareli and R. Ruckl, Phys. Lett. B144(1984)126; I. Bigi and S. Rudaz, Phys. Lett. 153(1985)335; M. Drees and M.M. Nojiri, Nucl. Phys. B396(1992)449.

[22] Jiang Yi, Han Liang, Ma Wen-Gan, Yu Zeng-Hui and Han Meng, J. of Phys. G23,385(1997), (Corrigendum G23, 1151(1997)); J. of Phys. G24, 83(1998).

[23] A.D. Martin, W.J. Stirling and R.G. Roberts, Phys. Lett.B354, 155(1995).

[24] J. Ellis and S. Rudaz, Phys. Lett. B128,248(1983);

[25] Bernd A. Kniehl, Phys. Rep. 240(1994)211.

[26] G. Passarino and M. Veltman, Nucl. Phys. B160151(1979). 
[27] 'The chargino pair production in proton-proton collisions', H. Zhou, W.G. Ma el al.,(in preparation).

\section{Figure Captions}

Fig.1 Feynman diagrams at one-loop level of the subprocess $g g \rightarrow \tilde{\chi}_{1}^{+} \tilde{\chi}_{1}^{-}$, where $(U, D)=$ $(u, d),(c, s),(t, b) . \quad(a .1 \sim a .3)$ box diagrams. $(b .1 \sim b .2)$ quartic interaction diagrams. $(c .1 \sim c .3)$ triangle interaction s-channel diagrams. The figures with exchanging incoming gluons in Fig.1(a) and Fig.1(c) are not shown.

Fig.2(a) The cross section $\hat{\sigma}$ of the higgsino-like chargino pair production subprocess $g g \rightarrow$ $\tilde{\chi}_{1}^{+} \tilde{\chi}_{1}^{-}$versus the c.m.s. energy of incoming gluons $\sqrt{\hat{s}}$ with $m_{\tilde{\chi}_{1}^{+}}=165 \mathrm{GeV}, m_{\tilde{\chi}_{2}^{+}}=750 \mathrm{GeV}$, $\tilde{M}=200 \mathrm{GeV}$ (for the third generation), $m_{A}=250 \mathrm{GeV}$, the masses of the first and second generation squarks being $600 \mathrm{GeV}$ and all vanishing $\mathrm{CP}$-odd phases. The full-line is for $\tan \beta=4$. The dashed-line for $\tan \beta=40$.

Fig.2(b) The cross section $\hat{\sigma}$ of the gaugino-like chargino pair production subprocess $g g \rightarrow$ $\tilde{\chi}_{1}^{+} \tilde{\chi}_{1}^{-}$versus the c.m.s. energy of incoming gluons $\sqrt{\hat{s}}$ with $m_{\tilde{\chi}_{1}^{+}}=165 \mathrm{GeV}, m_{\tilde{\chi}_{2}^{+}}=750 \mathrm{GeV}$, $m_{A}=250 \mathrm{GeV}, \tilde{M}=200 \mathrm{GeV}$ (for the third generation), the masses of the first and second generation squarks being $600 \mathrm{GeV}$ and all vanishing CP-odd phases. The full-line is for $\tan \beta=4$ and the dashed-line for $\tan \beta=40$.

Fig.3 The cross section $\hat{\sigma}$ of the higgsino-like chargino pair production subprocess $g g \rightarrow$ $\tilde{\chi}_{1}^{+} \tilde{\chi}_{1}^{-}$versus the lightest chargino mass with $\sqrt{\hat{s}}=450 \mathrm{GeV}, m_{\tilde{\chi}_{1}^{+}}=165 \mathrm{GeV}, m_{\tilde{\chi}_{2}^{+}}=$ $750 \mathrm{GeV}, m_{A}=250 \mathrm{GeV}, \tilde{M}=200 \mathrm{GeV}$ (for the third generation), the masses of the first 
and second generation squarks being $600 \mathrm{GeV}$ and all vanishing $\mathrm{CP}$-odd phases. The full-line is for $\tan \beta=4$ and the dashed-line for $\tan \beta=40$.

Fig.4 The cross section $\hat{\sigma}$ of the higgsino-like chargino pair production subprocess $g g \rightarrow$ $\tilde{\chi}_{1}^{+} \tilde{\chi}_{1}^{-}$versus the third generation $\tilde{M}\left(=M_{\tilde{Q}}=M_{\tilde{t}}=M_{\tilde{b}}\right)$ with $\sqrt{\hat{s}}=450 \mathrm{GeV}, m_{\tilde{\chi}_{1}^{+}}=$ $165 \mathrm{GeV}, m_{\tilde{\chi}_{2}^{+}}=750 \mathrm{GeV}, \phi_{\mu}=\phi_{\tilde{U}}=\phi_{\tilde{D}}=0, m_{A}=250 \mathrm{GeV}$ and all the masses of the first and second generation squarks being $600 \mathrm{GeV}$. The full-line is for $\tan \beta=4$ and the dashed-line for $\tan \beta=40$.

Fig.5(a) The cross section $\hat{\sigma}$ of the higgsino-like chargino pair production subprocess $g g \rightarrow$ $\tilde{\chi}_{1}^{+} \tilde{\chi}_{1}^{-}$versus $\phi_{C P}$ 's with $\sqrt{\hat{s}}=450 \mathrm{GeV}, m_{\tilde{\chi}_{1}^{+}}=165 \mathrm{GeV}, m_{\tilde{\chi}_{2}^{+}}=750 \mathrm{GeV}, \tan \beta=4$, $m_{A}=250 \mathrm{GeV}, \tilde{M}=200 \mathrm{GeV}$ (for the third generation) and the masses of the first and second generation squarks being $600 \mathrm{GeV}$. The full-line is for $\hat{\sigma}$ versus $\phi_{q}\left(=\phi_{\tilde{t}}=\phi_{\tilde{b}}\right)$. The dashed-line is for $\hat{\sigma}$ versus $\phi_{\mu}$.

Fig.5(b) The cross section $\hat{\sigma}$ of the higgsino-like chargino pair production subprocess $g g \rightarrow$ $\tilde{\chi}_{1}^{+} \tilde{\chi}_{1}^{-}$versus $\phi_{C P}$ 's with $\sqrt{\hat{s}}=450 \mathrm{GeV}, m_{\tilde{\chi}_{1}^{+}}=165 \mathrm{GeV}, m_{\tilde{\chi}_{2}^{+}}=750 \mathrm{GeV}, \tan \beta=40$, $m_{A}=250 \mathrm{GeV}, \tilde{M}=200 \mathrm{GeV}$ (for the third generation) and the masses of the first and second generation squarks being $600 \mathrm{GeV}$. The full-line is for $\hat{\sigma}$ versus $\phi_{q}\left(=\phi_{\tilde{t}}=\phi_{\tilde{b}}\right)$. The dashed-line is for $\hat{\sigma}$ versus $\phi_{\mu}$.

Fig.6 The cross section $\sigma$ of the higgsino-like chargino pair production process $p p \rightarrow g g \rightarrow$ $\tilde{\chi}_{1}^{+} \tilde{\chi}_{1}^{-}+X$ versus the c.m.s energy of proton-proton collider $\sqrt{s}$. We take $m_{\tilde{\chi}_{1}^{+}}=165 \mathrm{GeV}$, $m_{\tilde{\chi}_{2}^{+}}=750 \mathrm{GeV}, m_{A}=250 \mathrm{GeV}, \tilde{M}=200 \mathrm{GeV}$ (for the third generation) and the masses of the first and second generation squarks being $600 \mathrm{GeV}$. The full-line is for $\tan \beta=4$ with all 
vanishing $\mathrm{CP}$-odd phases, the dashed-line for $\tan \beta=40$ with all vanishing $\mathrm{CP}$-odd phases and the dotted-line for the case with $\tan \beta=40, \phi_{t}=\phi_{b}=\pi / 4$ and other phase angels being zero. 


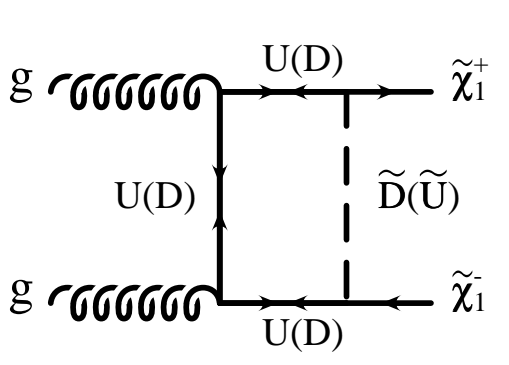

(a.1)

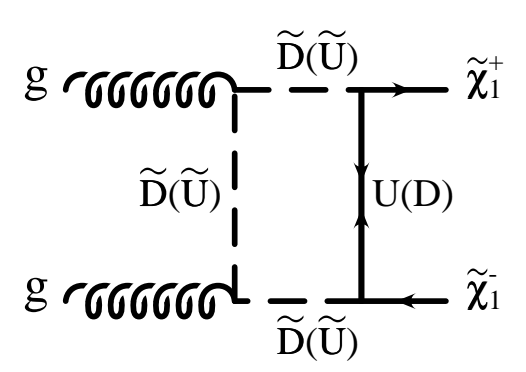

(a.2)

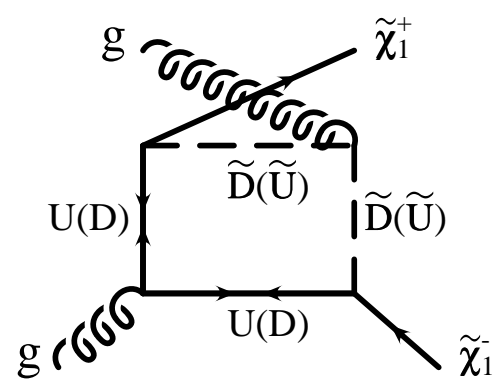

(a.3)

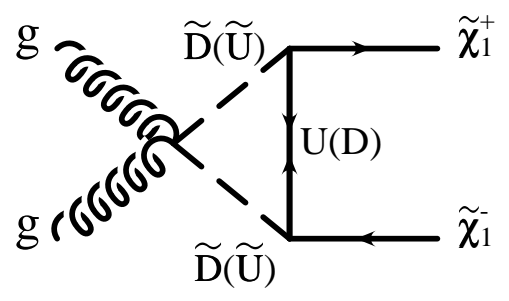

(b.1)

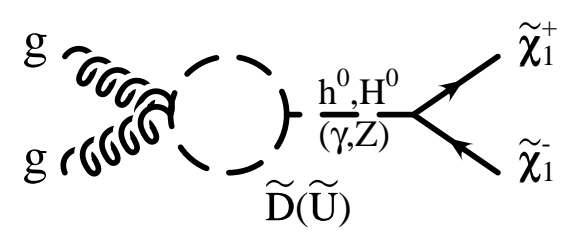

(b.2)

(c.1)

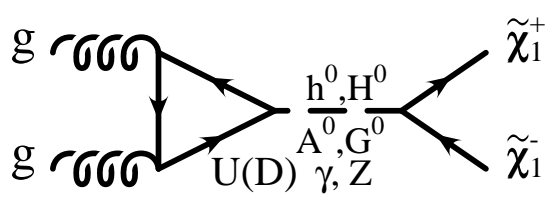

(c.2)

Fig.1 
Fig.2(a)

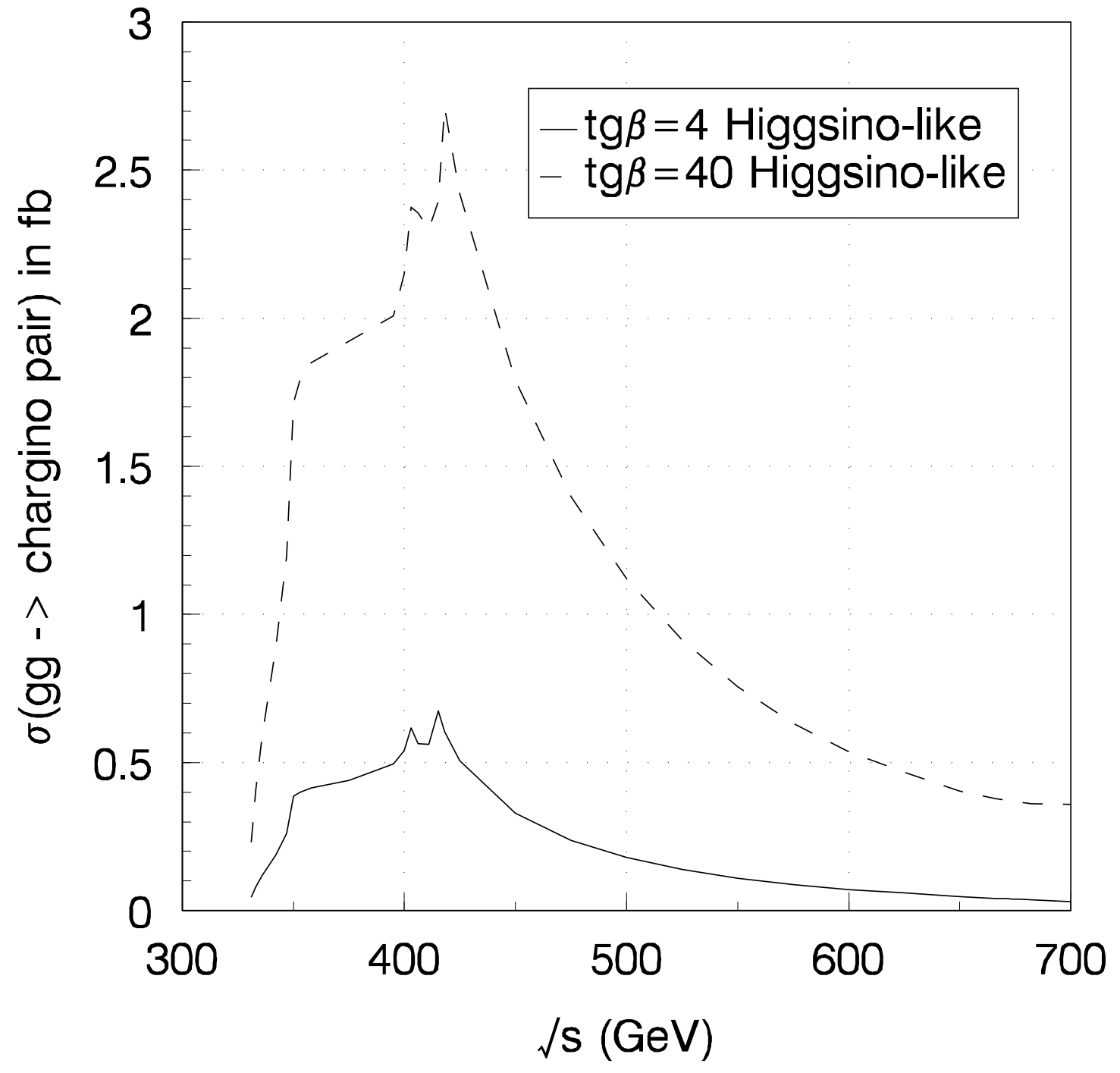


Fig.2(b)

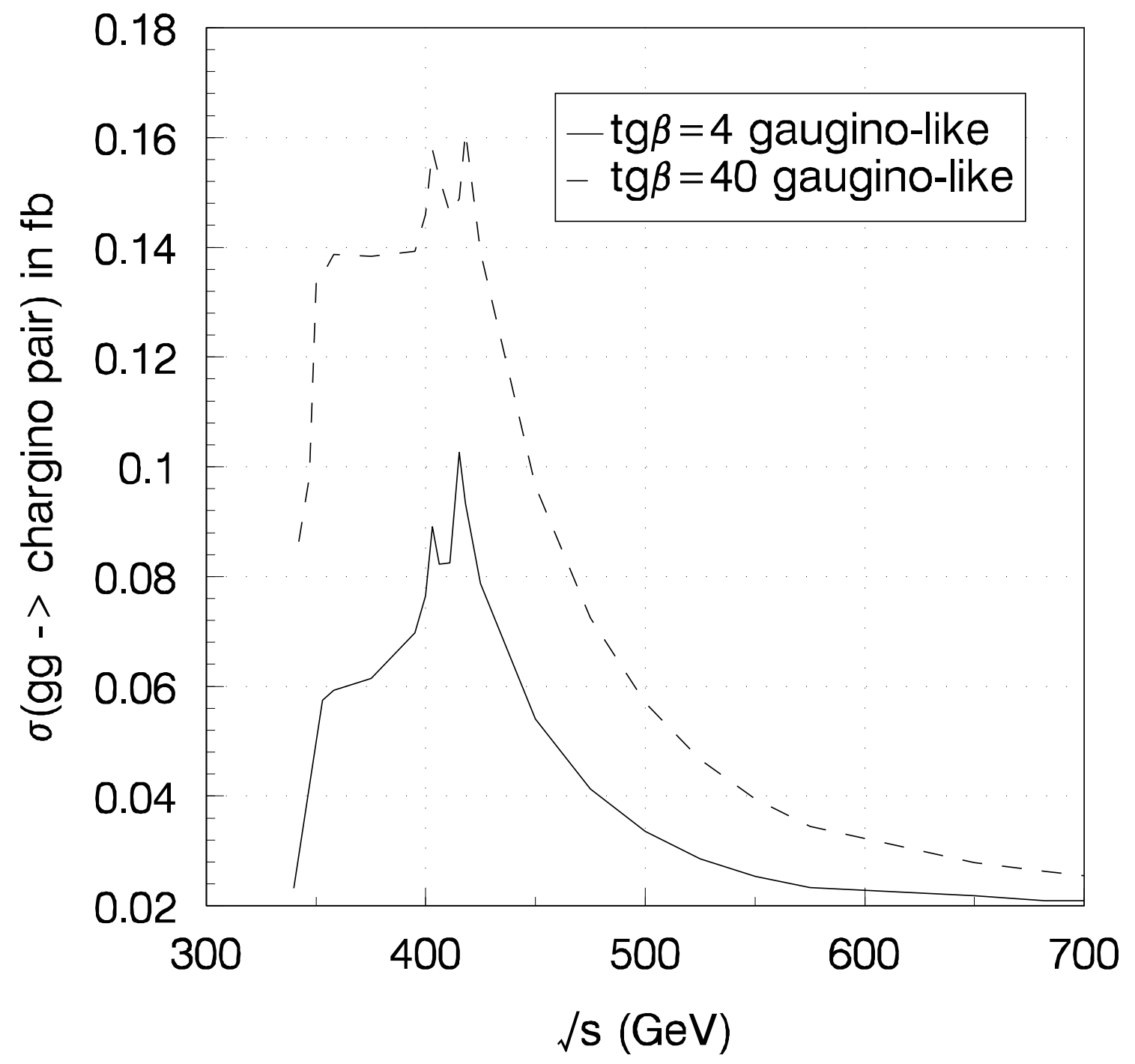


Fig.3

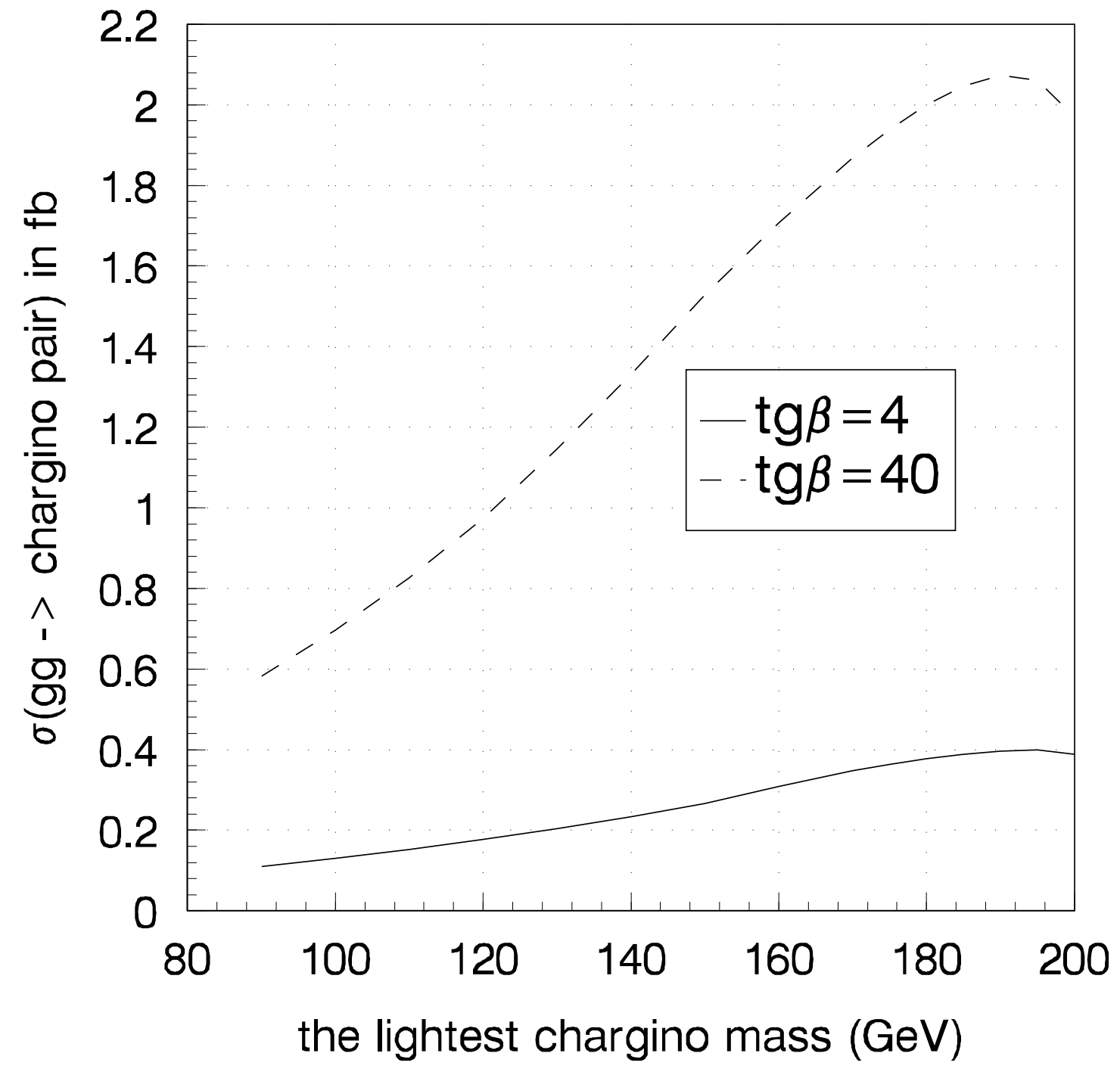


Fig.4

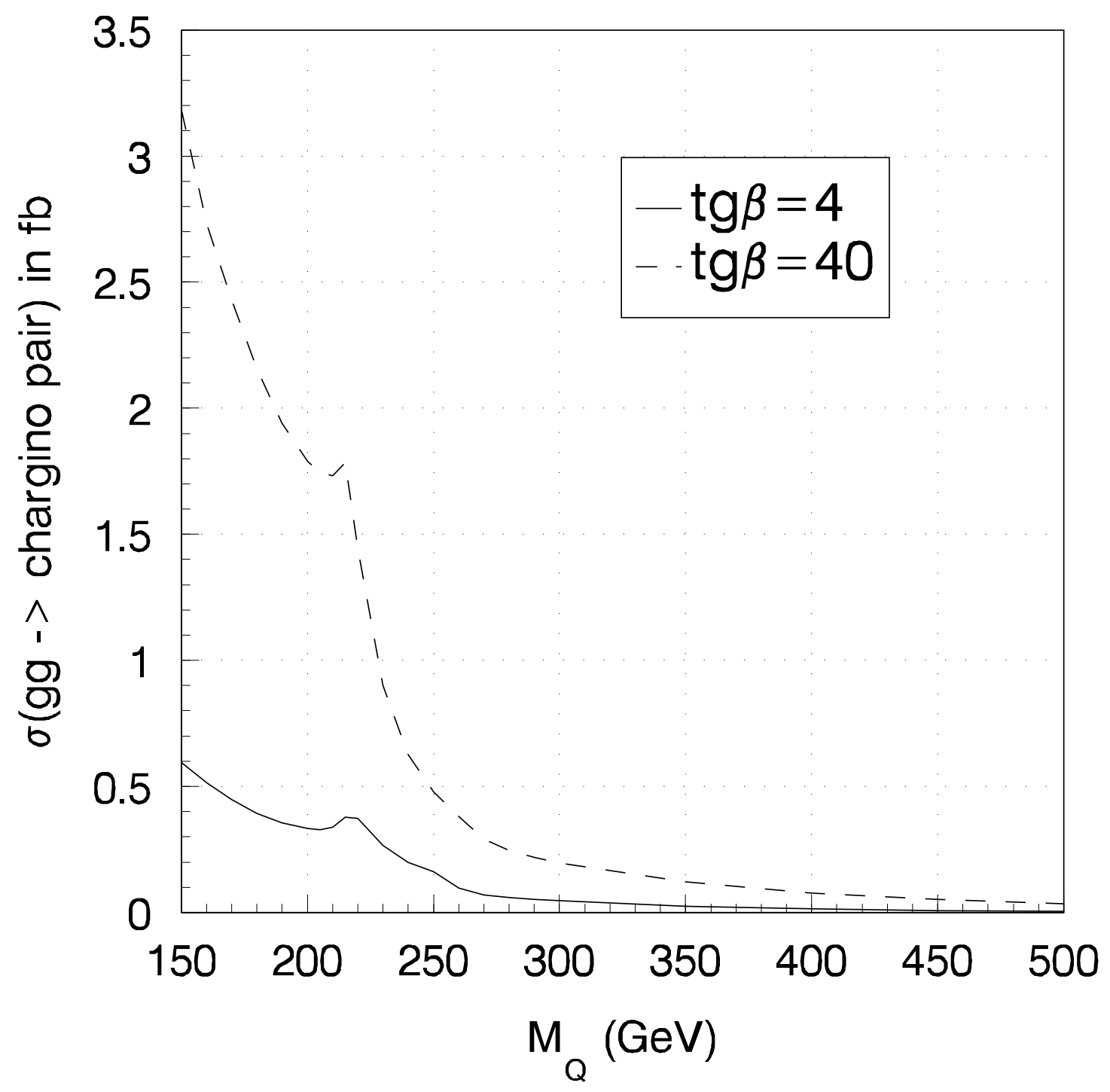


Fig.5(a)

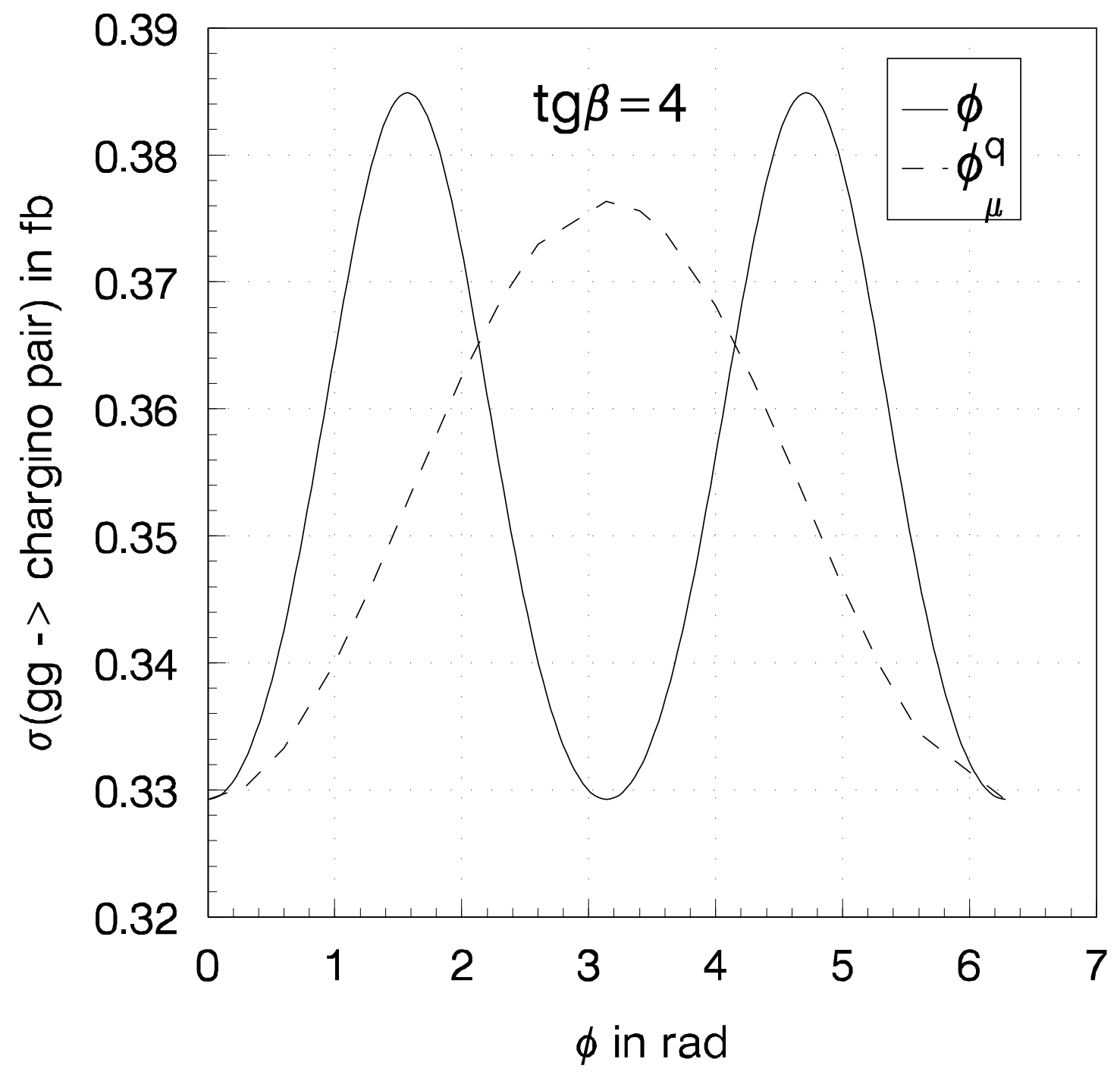


Fig.5(b)

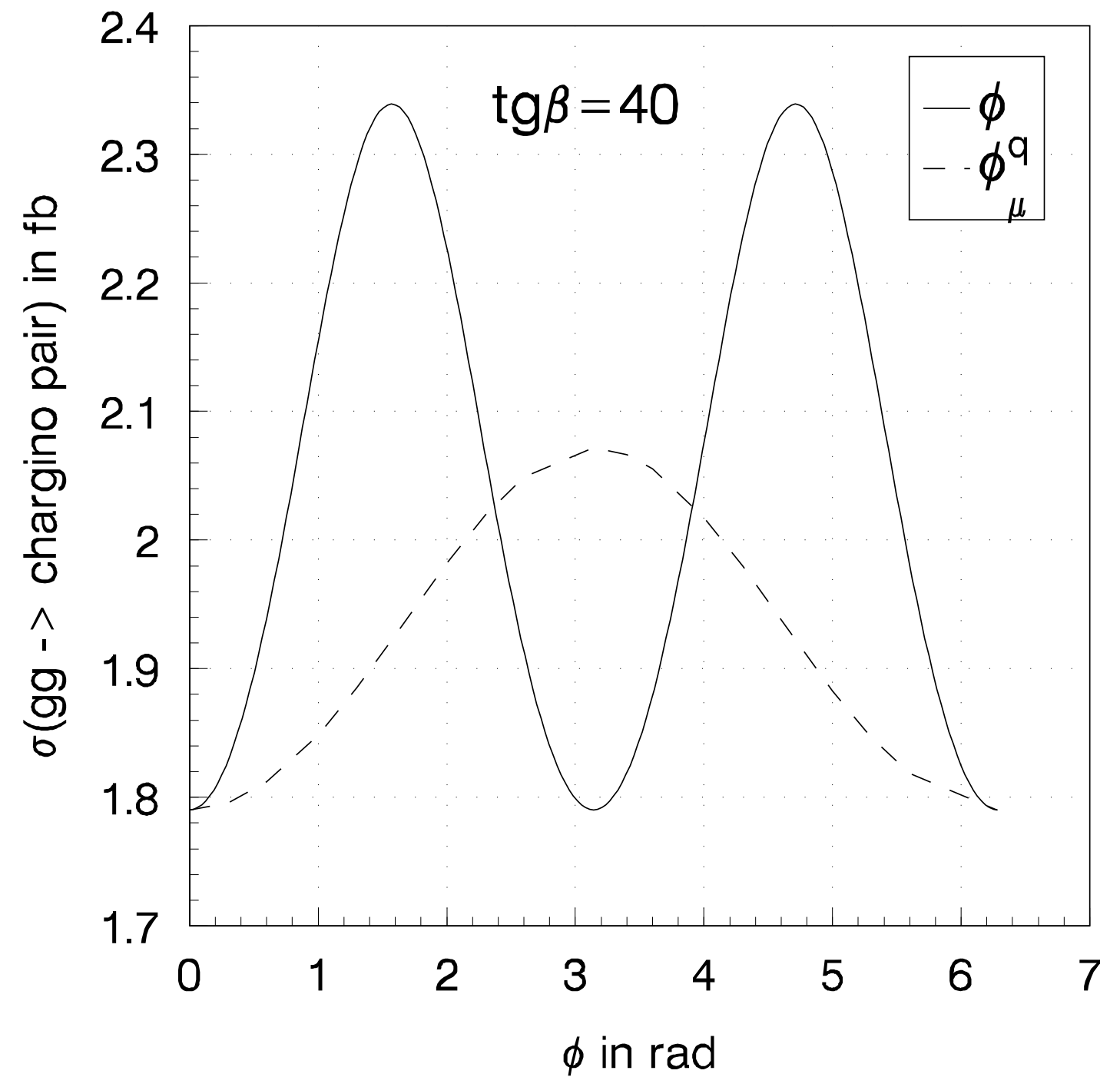


Fig.6

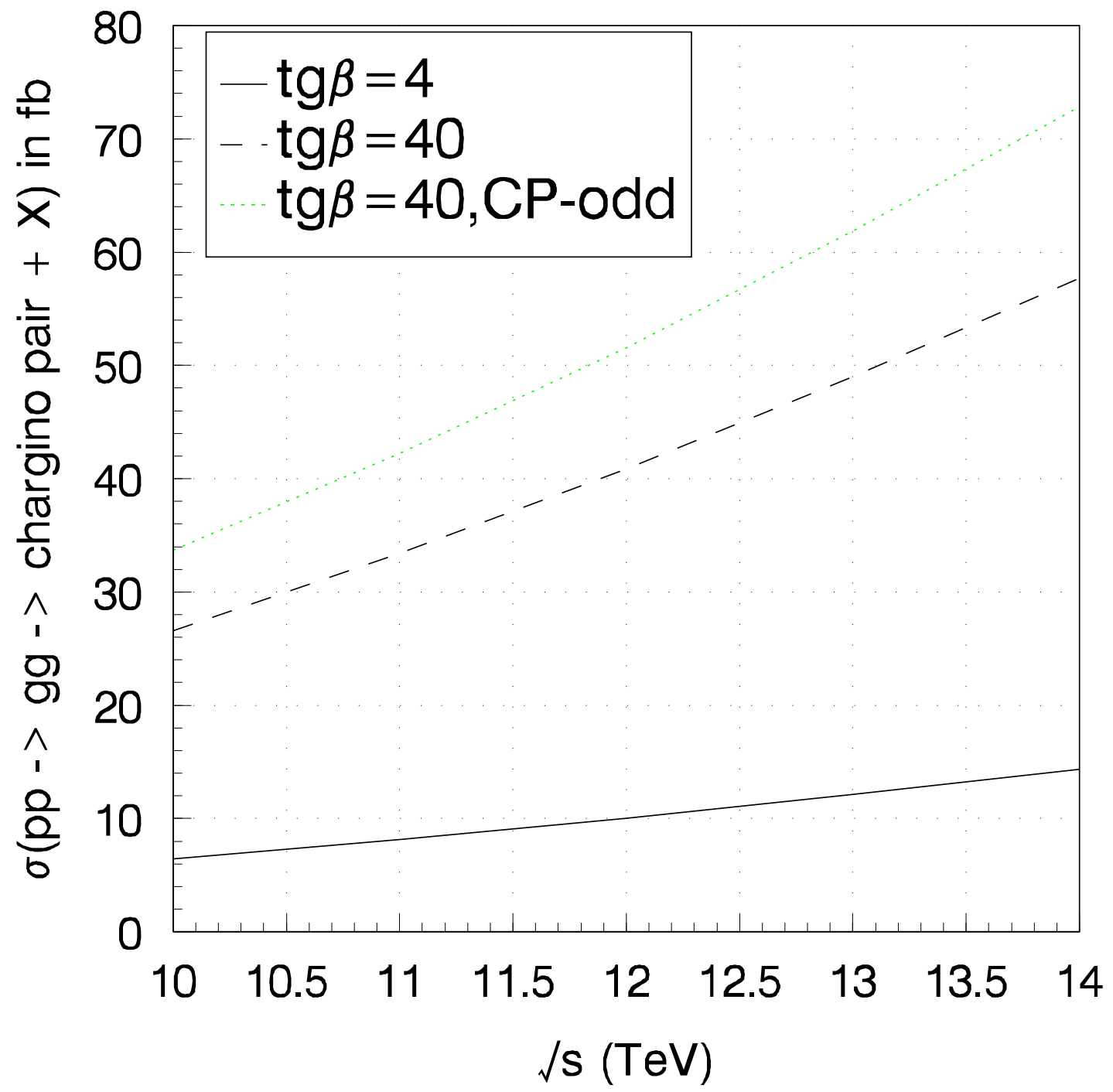

